\title{
Approximate Controllability for Impulsive Riemann-Liouville Fractional Differential Inclusions
}

\author{
Zhenhai Liu and Maojun Bin \\ Guangxi Key Laboratory of Hybrid Computation and IC Design Analysis, College of Sciences, Guangxi University for Nationalities, \\ Nanning, Guangxi 530006, China
}

Correspondence should be addressed to Zhenhai Liu; zhhliu@hotmail.com

Received 21 July 2013; Revised 25 September 2013; Accepted 2 November 2013

Academic Editor: Naseer Shahzad

Copyright (C) 2013 Z. Liu and M. Bin. This is an open access article distributed under the Creative Commons Attribution License, which permits unrestricted use, distribution, and reproduction in any medium, provided the original work is properly cited.

\begin{abstract}
We study the control systems governed by impulsive Riemann-Liouville fractional differential inclusions and their approximate controllability in Banach space. Firstly, we introduce the $P C_{1-\alpha}$-mild solutions for the impulsive Riemann-Liouville fractional differential inclusions in Banach spaces. Secondly, by using the fractional power of operators and a fixed point theorem for multivalued maps, we establish sufficient conditions for the approximate controllability for a class of Riemann-Liouville fractional impulsive differential inclusions, which is a generalization and continuation of the recent results on this issue. At the end, we give an example to illustrate the application of the abstract results.
\end{abstract}

\section{Introduction}

The concept of controllability plays an important part in the analysis and design of control systems. Since Kalman [1] first introduced its definition in 1963, controllability of the deterministic and stochastic dynamical control systems in finite-dimensional and infinite-dimensional spaces is well developed in different classes of approaches, and more details can be found in papers [2-4]. Some authors [5-7] have studied the exact controllability for nonlinear evolution systems by using the fixed point theorems. In [5-7], to prove the controllability results for fractional-order semilinear systems, the authors made an assumption that the semigroup associated with the linear part is compact. But if $C_{0}$-semigroup $T(t)$ is compact or the operator $B$ is compact, then the controllability operator is also compact and hence the inverse of it does not exist if the state space $V$ is infinite dimensional [8]. Thus, it is shown that the concept of exact controllability is difficult to be satisfied in infinite-dimensional space. Therefore, it is important to study the weaker concept of controllability, namely, approximate controllability for differential equations. In these years, several researchers [9-17] have studied it for control systems.
In [13], Sakthivel et al. studied on the approximate controllability of semilinear fractional differential systems:

$$
\begin{gathered}
{ }^{c} D^{\alpha} x(t)=A x(t)+B u(t)+f(t, x(t)), \quad t \in J=[0, T], \\
x(0)=x_{0},
\end{gathered}
$$

where ${ }^{c} D_{t}^{\alpha}$ is Caputo's fractional derivative of $0<\alpha<1$ and $A$ is the infinitesimal generator of a $C_{0}$-semigroup $T(t)$ of bounded operators on the Hilbert space $X$; the control function $u(\cdot)$ is given in $L^{2}(J, U) ; U$ is a Hilbert space; $B$ is a bounded linear operator from $U$ to $X ; f: J \times X \rightarrow X$ is a given function satisfying some assumptions and $x_{0}$ is an element of the Hilbert space $X$.

In [16], Sukavanam and Kumar researched approximate controllability of fractional-order semilinear delay systems:

$$
\begin{gathered}
\frac{d^{\alpha} x(t)}{t^{\alpha}}=A x(t)+B u(t)+f\left(t, x_{t}, u(t)\right), \quad t \in[0, \tau], \\
x_{0}(\theta)=\phi(\theta), \quad \theta \in[-h, 0],
\end{gathered}
$$

where $1 / 2<\alpha<1 ; A: D(A) \subseteq V \rightarrow V$ is a closed linear operator with dense domain $D(A)$ generating 
a $C_{0}$-semigroup $S(t)$; the state $x(\cdot)$ takes values in the Banach space $V$; the control function $u(\cdot)$ takes values in $\widehat{V} ; B$ is a bounded linear operator from $L^{2}([0, \tau] ; \widehat{V})$ to $L^{2}([0, \tau] ; V)$; the operator $f:[0, \tau] \times C([-h, 0] ; V) \times \widehat{V} \rightarrow V$ is nonlinear. If $x:[-h, \tau] \rightarrow V$ is a continuous function, then $x_{t}:$ $[-h, 0] \rightarrow V$ is defined as $x_{t}(\theta)=x(t+\theta)$ for $\theta \in[-h, 0]$ and $\phi \in C([-h, 0] ; V)$.

In [18], Rykaczewski studied the approximate controllability of an inclusion of the form

$$
\begin{gathered}
\dot{x}(t) \in A x(t)+F(t, x(t))+B u(t), \quad t \in J=[0, b], \\
x(0)=x_{0},
\end{gathered}
$$

where $A$ is a linear operator which generates a compact semigroup, $F$ is u.h.c. multivalued perturbation with weakly compact values, and the state $x(\cdot)$ takes values in the Hilbert space $H . U$ is a Hilbert space of all admissible controls. $B$ : $U \rightarrow H$ is a continuous linear operator.

Fractional differential equations have recently proved to be valuable tools in the modeling of many phenomena in various fields of engineering, physics, and economics. Indeed, we can find numerous applications in viscoelasticity, electrochemistry, control, porous media, electromagnetic, and so forth; see [19-27] for example. As a consequence there was an intensive development of the theory of differential equations of fractional order. One can see the monographs of Kilbas et al. [28] and Podlubny [29] and the references therein. The definitions of Riemann-Liouville fractional derivatives or integrals with initial conditions are strong tools to resolve some fractional differential problems in the real world. Heymans and Podlubny [30] have verified that it was possible to attribute physical meaning to initial conditions expressed in terms of Riemann-Liouville fractional derivatives or integrals, and such initial conditions are more appropriate than physically interpretable initial conditions. Furthermore, they have investigated that the impulse response with RiemannLiouville fractional derivatives was seldom used in the fields of physics, such as viscoelasticity. In recent years, many authors $[18,27,31]$ were devoted to mild solutions to fractional evolution equations with Caputo fractional derivative, and there have been a lot of interesting works. As for the study of the fractional differential systems with Caputo fractional derivative, we can refer to $[27,31,32]$ for the existence results. Its approximate controllability was considered in [9, 13-16]. The approximate controllability of Caputo fractional inclusion systems has been investigated by [10]. We know that differential inclusions are strong tools to solve some problems in various fields of engineering, physics, and optimal control; see $[10,32-35]$. However, the approximate controllability for the impulsive fractional differential evolution inclusion with Riemann-Liouville fractional derivatives is still an untreated topic in the literature.

Motivated by the above work, in this paper, we consider the following system:

$$
\begin{array}{r}
D_{t}^{\alpha} x(t) \in A x(t)+F(t, x(t))+B u(t), \\
t \in J^{\prime}=(0, b] \backslash\left\{t_{1}, t_{2}, \ldots, t_{m}\right\},
\end{array}
$$

$$
\begin{gathered}
\left.\Delta I_{0^{+}}^{1-\alpha} x(t)\right|_{t=t_{k}}=I_{k}\left(x\left(t_{k}^{-}\right)\right), \quad k=1,2, \ldots, m, \\
\left.I_{t}^{1-\alpha} x(t)\right|_{t=0}=x_{0} \in X,
\end{gathered}
$$

where $1 / 2<\alpha \leq 1$ and $D_{t}^{\alpha}$ denotes the Riemann-Liouville fractional derivative of order $\alpha$ with the lower limit zero. $F$ : $J \times X \rightarrow \mathscr{P}(X):=2^{X} \backslash\{\emptyset\}$ is a nonempty, bounded, closed, and convex multivalued map. $A: D(A) \subseteq X \rightarrow X$ is the infinitesimal generator of a $C_{0}$-semigroup $T(t)(t \geq 0)$ on a Banach space $X .0=t_{0}<t_{1}<\cdots<t_{m}<t_{m+1}=b, I_{k}$ : $X \mapsto X, \Delta I_{0^{+}}^{1-\alpha} x\left(t_{k}\right)=I_{0^{+}}^{1-\alpha} x\left(t_{k}^{+}\right)-I_{0^{+}}^{1-\alpha} x\left(t_{k}^{-}\right), I_{0^{+}}^{1-\alpha} x\left(t_{k}^{+}\right)$and $I_{0^{+}}^{1-\alpha} x\left(t_{k}^{-}\right)$denote the right and the left limits of $I_{0^{+}}^{1-\alpha} x(t)$ at $t=t_{k}, k=1,2, \ldots, m$. The control function $u(t)$ takes value in $V=L^{p}([0, b] ; U), p>1 / \alpha$, and $U$ is a Banach space; $B$ is a linear operator from $V$ to $L^{p}([0, b] ; X)$.

The purpose of this paper is to provide some suitable sufficient conditions for the existence of mild solutions and approximate controllability results for the impulsive fractional abstract Cauchy problems with Riemann-Liouville fractional derivatives. The main tools used in our study are fixed point theorem, semigroup theory for multivalued maps, and the theory from fractional differential equations. The rest of this paper is organized as follows. In Section 2, we present some preliminaries to prove our main results. In Section 3, by applying some standard fixed point principles, we prove the existence of the mild solutions for semilinear fractional differential equations, and the approximate controllability of the system (4) is proved. In Section 4, we give an example to illustrate our main results.

\section{Preliminaries}

In this section, we introduce some basic definitions and preliminaries which are used throughout this paper. The norm of a Banach space $X$ will be denoted by $\|\cdot\|_{X} \cdot L_{b}(X, Y)$ denotes the space of bounded linear operators from $X$ to $Y$. For the uniformly bounded $C_{0}$-semigroup $T(t)(t \geq 0)$, we set $M:=\sup _{t \in[0, \infty)}\|T(t)\|_{L_{b}(X)}<\infty$. Let $C(J, X)$ denote the Banach space of all $X$ value continuous functions from $J=[0, b]$ to $X$ with the norm $\|x\|_{C}=\sup _{t \in J}\|x(t)\|_{X}$. Let $C_{1-\alpha}(J, X)=\left\{x: t^{1-\alpha} x(t) \in C(J, X)\right\}$ with the norm

$$
\|x\|_{C_{1-\alpha}}=\sup \left\{t^{1-\alpha}\|x(t)\|_{X}: t \in J\right\} .
$$

Obviously, the space $C_{1-\alpha}(J, X)$ is a Banach space.

To define the mild solutions of (4), we also consider the Banach space $P C_{1-\alpha}(J, X)=\left\{x:\left(t-t_{k}\right)^{1-\alpha} x(t) \epsilon\right.$ $C\left(\left(t_{k}, t_{k+1}\right], X\right), \lim _{t \rightarrow t_{k}^{+}}\left(t-t_{k}\right)^{1-\alpha} x(t)$ exist, and $x\left(t_{k}^{-}\right)$with $\left.x\left(t_{k}^{-}\right)=x\left(t_{k}\right), k=0,1,2, \ldots, m\right\}$ with the norm

$$
\begin{gathered}
\|x\|_{P C_{1-\alpha}}=\max \left\{\sup \left(t-t_{k}\right)^{1-\alpha}\|x(t)\|_{X}: t \in\left(t_{k}, t_{k+1}\right],\right. \\
k=0,1,2, \ldots, m\} .
\end{gathered}
$$

It is easily known that the space $P C_{1-\alpha}(J, X)$ is a Banach space. 
Let $(X, d)$ be a metric space. We use the notations

$$
\begin{gathered}
P_{c l}(X)=\{Y \in \mathscr{P}(X): Y \text { is closed }\} \\
P_{b}(X)=\{Y \in \mathscr{P}(X): Y \text { is bounded }\}, \\
P_{c v}(X)=\{Y \in \mathscr{P}(X): Y \text { is convex }\} \\
P_{c p}(X)=\{Y \in \mathscr{P}(X): Y \text { is compact }\} .
\end{gathered}
$$

Firstly, let us recall the following basic definitions from fractional calculus. For more details, one can see $[28,29]$.

Definition 1. The integral

$$
I_{t}^{\alpha} f(t)=\frac{1}{\Gamma(\alpha)} \int_{0}^{t}(t-s)^{\alpha-1} f(s) d s, \quad \alpha>0,
$$

is called Riemann-Liouville fractional integral of order $\alpha$, where $\Gamma$ is the gamma function.

Definition 2. For a function $f(t)$ given in the interval $[0, \infty)$, the expression

$$
D_{t}^{\alpha} f(t)=\frac{1}{\Gamma(n-\alpha)}\left(\frac{d}{d t}\right)^{n} \int_{0}^{t}(t-s)^{n-\alpha-1} f(s) d t,
$$

where $n=[\alpha]+1$ and $[\alpha]$ denotes the integer part of number $\alpha$, is called the Riemann-Liouville fractional derivative of order $\alpha$.

Lemma 3 (see [28]). Let $\alpha>0, m=[\alpha]+1$, and let $x_{m-\alpha}(t)=$ $I_{t}^{m-\alpha} x(t)$ be the fractional integral of order $m-\alpha$. If $x(t) \in$ $L^{1}(J, X)$ and $x_{m-\alpha}(t) \in A C^{m}(J, X)$, then one has the following equality:

$$
I_{t}^{\alpha} D_{t}^{\alpha} x(t)=x(t)-\sum_{k=1}^{m} \frac{x_{m-\alpha}^{(m-k)}(0)}{\Gamma(\alpha-k+1)} t^{\alpha-k}
$$

In order to study the $P C$-mild solutions of (4) in Banach space $P C_{1-\alpha}(J, X)$, we give the following results which will be used throughout this paper.

Lemma 4. Let $0<\alpha \leq 1$, and let $x_{1-\alpha}(t)=I_{t}^{1-\alpha} x(t)$ be the fractional integral of order $1-\alpha$. If $x(t) \in P C_{1-\alpha}(J, X)$ and $x_{1-\alpha}(t) \in P C(J, X)$, then one has the following equality:

$$
\begin{aligned}
& I_{t}^{\alpha} D_{t}^{\alpha} x(t) \\
& =\left\{\begin{array}{cc}
x(t)-\left.x_{1-\alpha}(t)\right|_{t=0} \frac{t^{\alpha-1}}{\Gamma(\alpha)}, & t \in\left[0, t_{1}\right], \\
x(t)-\sum_{k=1}^{m} \frac{\Delta x_{1-\alpha}\left(t_{i}\right)}{\Gamma(\alpha)}\left(t-t_{i}\right)^{\alpha-1} & \\
-\left.x_{1-\alpha}(t)\right|_{t=0} \frac{t^{\alpha-1}}{\Gamma(\alpha)}, & t \in\left(t_{k}, t_{k+1}\right],
\end{array}\right.
\end{aligned}
$$

Proof. If $t \in\left[0, t_{1}\right]$, then, by Lemma 3, we easily get

$$
I_{0^{+}}^{\alpha} D_{0^{+}}^{\alpha} x(t)=x(t)-\left.x_{1-\alpha}(t)\right|_{t=0} \frac{t^{\alpha-1}}{\Gamma(\alpha)} .
$$

If $t \in\left(t_{1}, t_{2}\right]$, since

$$
\begin{aligned}
I_{t}^{\alpha} D_{t}^{\alpha} x(t) & =\frac{1}{\Gamma(\alpha)} \int_{0}^{t}(t-s)^{\alpha-1} D_{t}^{\alpha} x(s) d s \\
& =\frac{d}{d t}\left\{\frac{1}{\Gamma(\alpha+1)} \int_{0}^{t}(t-s)^{\alpha L} D_{t}^{\alpha} x(s) d s\right\},
\end{aligned}
$$

then, by (13), we have

$$
\begin{aligned}
& \frac{1}{\Gamma(\alpha+1)} \int_{0}^{t}(t-s)^{\alpha L} D_{t}^{\alpha} x(s) d s \\
& =\frac{1}{\Gamma(\alpha+1)} \int_{0}^{t}(t-s)^{\alpha} \frac{d}{d s}\left\{I_{0^{+}}^{1-\alpha} x(s)\right\} d s \\
& =\frac{1}{\Gamma(\alpha+1) \Gamma(1-\alpha)} \\
& \times \int_{0}^{t}(t-s)^{\alpha} \frac{d}{d s} \int_{0}^{s}(s-\tau)^{1-\alpha-1} x(\tau) d \tau d s \\
& =\frac{1}{\Gamma(\alpha+1) \Gamma(1-\alpha)} \\
& \times \int_{0}^{t_{1}}(t-s)^{\alpha} \frac{d}{d s} \int_{0}^{s}(s-\tau)^{1-\alpha-1} x(\tau) d \tau d s \\
& +\frac{1}{\Gamma(\alpha+1) \Gamma(1-\alpha)} \\
& \times \int_{t_{1}}^{t}(t-s)^{\alpha} \frac{d}{d s} \int_{0}^{t_{1}}(s-\tau)^{1-\alpha-1} x(\tau) d \tau d s \\
& +\frac{1}{\Gamma(\alpha+1) \Gamma(1-\alpha)} \\
& \times \int_{t_{1}}^{t}(t-s)^{\alpha} \frac{d}{d s} \int_{t_{1}}^{s}(s-\tau)^{1-\alpha-1} x(\tau) d \tau d s \\
& =\frac{1}{\Gamma(\alpha) \Gamma(1-\alpha)} \\
& \times \int_{0}^{t_{1}}(t-s)^{\alpha-1} \int_{0}^{s}(s-\tau)^{1-\alpha-1} x(\tau) d \tau d s \\
& +\frac{1}{\Gamma(\alpha+1) \Gamma(1-\alpha)} \\
& \times\left.\left[(t-s)^{\alpha} \int_{0}^{s}(s-\tau)^{1-\alpha-1} x(\tau) d \tau\right]\right|_{s=0} ^{s=t_{1}} \\
& +\frac{1}{\Gamma(\alpha) \Gamma(1-\alpha)} \\
& \times \int_{t_{1}}^{t}(t-s)^{\alpha-1} \int_{0}^{t_{1}}(s-\tau)^{1-\alpha-1} x(\tau) d \tau d s \\
& +\frac{1}{\Gamma(\alpha+1) \Gamma(1-\alpha)}
\end{aligned}
$$




$$
\begin{aligned}
& \times\left.\left[(t-s)^{\alpha} \int_{0}^{t_{1}}(s-\tau)^{1-\alpha-1} x(\tau) d \tau\right]\right|_{s=t_{1}} ^{s=t} \\
& +\frac{1}{\Gamma(\alpha) \Gamma(1-\alpha)} \\
& \times \int_{t_{1}}^{t}(t-s)^{\alpha-1} \int_{t_{1}}^{s}(s-\tau)^{1-\alpha-1} x(\tau) d \tau d s \\
& +\frac{1}{\Gamma(\alpha+1) \Gamma(1-\alpha)} \\
& \times\left.\left[(t-s)^{\alpha} \int_{t_{1}}^{s}(s-\tau)^{1-\alpha-1} x(\tau) d \tau\right]\right|_{s=t_{1}} ^{s=t} \\
& =\frac{1}{\Gamma(\alpha) \Gamma(1-\alpha)} \\
& \times \int_{0}^{t_{1}} x(\tau) d \tau \int_{\tau}^{t_{1}}(t-s)^{\alpha-1}(s-\tau)^{1-\alpha-1} d s \\
& +\frac{1}{\Gamma(\alpha) \Gamma(1-\alpha)} \\
& \times \int_{0}^{t_{1}} x(\tau) d \tau \int_{t_{1}}^{t}(t-s)^{\alpha-1}(s-\tau)^{1-\alpha-1} d s \\
& +\frac{1}{\Gamma(\alpha) \Gamma(1-\alpha)} \\
& \times \int_{t_{1}}^{t} x(\tau) d \tau \int_{\tau}^{t}(t-s)^{\alpha-1}(s-\tau)^{1-\alpha-1} d s \\
& -\frac{x_{1-\alpha}(0)}{\Gamma(\alpha+1)} t^{\alpha}-\frac{\Delta x_{1-\alpha}\left(t_{1}\right)}{\Gamma(\alpha+1)}\left(t-t_{1}\right)^{\alpha} \\
& =\int_{0}^{t} x(\tau) d \tau-\frac{x_{1-\alpha}(0)}{\Gamma(\alpha+1)} t^{\alpha}-\frac{\Delta x_{1-\alpha}\left(t_{1}\right)}{\Gamma(\alpha+1)}\left(t-t_{1}\right)^{\alpha} .
\end{aligned}
$$

Thus, by (13) and (14), we get

$$
I_{t}^{\alpha} D_{t}^{\alpha} x(t)=x(t)-\frac{x_{1-\alpha}(0)}{\Gamma(\alpha)} t^{\alpha-1}-\frac{\Delta x_{1-\alpha}\left(t_{1}\right)}{\Gamma(\alpha)}\left(t-t_{1}\right)^{\alpha-1}
$$

Similarly, if $t \in\left(t_{k}, t_{k+1}\right], k=2, \ldots, m$, we can get

$$
\begin{aligned}
& I_{t}^{\alpha} D_{t}^{\alpha} x(t) \\
& =x(t)-\sum_{k=1}^{m} \frac{\Delta x_{1-\alpha}\left(t_{k}\right)}{\Gamma(\alpha)}\left(t-t_{k}\right)^{\alpha-1}-\left.x_{1-\alpha}(t)\right|_{t=0} \frac{t^{\alpha-1}}{\Gamma(\alpha)} .
\end{aligned}
$$

The proof is completed.

The Laplace transform formula for the Riemann-Liouville fractional integral is defined by

$$
L\left\{I_{t}^{\alpha} x(t) ; \lambda\right\}=\frac{1}{\lambda^{\alpha}} \widehat{x}(\lambda)
$$

where $\widehat{x}(\lambda)$ is the Laplace of $x$ defined by

$$
\widehat{x}(\lambda)=\int_{0}^{\infty} e^{-\lambda t} x(t) d t
$$

$$
\operatorname{Re} \lambda>\omega, \quad|x(t)| \leq c e^{\omega t}, \quad c \text { is a constant. }
$$

Lemma 5. Let $\alpha \in(0,1]$ and $h \in L^{p}(J, X), p>1 / \alpha$; if $x(t) \epsilon$ $P C_{1-\alpha}(J, X), x_{1-\alpha}(t) \in P C(J, X)$, and $x$ is a solution of the following problem:

$$
\begin{gathered}
D_{t}^{\alpha} x(t)=A x(t)+h(t), \\
t \in(0, b], \quad t \neq t_{k}, \quad k=1,2, \ldots, m, \\
\left.\Delta I_{t}^{1-\alpha} x(t)\right|_{t=t_{k}}=I_{k}\left(x\left(t_{k}^{-}\right)\right), \quad k=1,2, \ldots, m, \\
\left.I_{t}^{1-\alpha} x(t)\right|_{t=0}=x_{0} \in X,
\end{gathered}
$$

then, $x$ satisfies the following equation:

$x(t)$

$$
=\left\{\begin{array}{c}
t^{\alpha-1} T_{\alpha}(t) x_{0}+\int_{0}^{t}(t-s)^{\alpha-1} T_{\alpha}(t-s) h(s) d s, \\
t \in\left[0, t_{1}\right], \\
t^{\alpha-1} T_{\alpha}(t) x_{0}+\sum_{k=1}^{m} T_{\alpha}\left(t-t_{k}\right)\left(t-t_{k}\right)^{\alpha-1} \Delta I_{0^{+}}^{1-\alpha} x\left(t_{k}\right) \\
+\int_{0}^{t}(t-s)^{\alpha-1} T_{\alpha}(t-s) h(s) d s, \\
t \in\left(t_{k}, t_{k+1}\right],
\end{array}\right.
$$

where $k=1,2, \ldots, m$,

$$
\begin{gathered}
T_{\alpha}(t)=\alpha \int_{0}^{\infty} \theta \xi_{\alpha}(\theta) T\left(t^{\alpha} \theta\right) d \theta, \\
\xi_{\alpha}(\theta)=\frac{1}{\alpha} \theta^{-1-(1 / \alpha)} \omega_{\alpha}\left(\theta^{-1 / \alpha}\right), \\
\omega_{\alpha}(\theta)=\frac{1}{\pi} \sum_{n=1}^{\infty}(-1)^{n-1} \theta^{-n \alpha-1} \frac{\Gamma(n \alpha+1)}{n !} \sin (n \pi \alpha), \\
\theta \in(0, \infty),
\end{gathered}
$$

where $\xi_{\alpha}$ is a probability density function defined on $(0, \infty)$; that is,

$$
\xi_{\alpha}(\theta) \geq 0, \quad \theta \in(0, \infty), \quad \int_{0}^{\infty} \xi_{\alpha}(\theta) d \theta=1
$$

Proof. We observe that $x(\cdot)$ can be decomposed to $p(\cdot)+q(\cdot)$, where $p$ is the continuous mild solution for

$$
\begin{gathered}
D_{t}^{\alpha} p(t)=A p(t)+h(t), \quad t \in(0, b], \\
\left.I_{t}^{1-\alpha} p(t)\right|_{t=0}=x_{0} \in X,
\end{gathered}
$$


and $q$ is the $P C_{1-\alpha}$-mild solution for

$$
\begin{gathered}
D_{t}^{\alpha} q(t)=A q(t), \\
t \in(0, b], \quad t \neq t_{k}, \quad k=1,2, \ldots, m, \\
\left.\Delta I_{t}^{1-\alpha} q(t)\right|_{t=t_{k}}=y_{k}, \quad k=1,2, \ldots, m, \\
\left.I_{t}^{1-\alpha} q(t)\right|_{t=0}=0 \in X .
\end{gathered}
$$

Indeed, by adding together (23) and (24), it follows by (19). Since $p$ is continuous, then $p\left(t_{k}^{+}\right)=p\left(t_{k}^{-}\right), k=1,2, \ldots, m$. On the other hand, any solution of (19) can be decomposed to (23) and (24). So we show the results by the following.

At first, we calculate the mild solution of (23).

Apply Riemann-Liouville fractional integral operator on both sides of (23); then, by Lemma 3, we get

$$
\begin{aligned}
p(t) & =\frac{\left.I_{t}^{1-\alpha} p(t)\right|_{t=0}}{\Gamma(\alpha)} t^{\alpha-1}+I_{t}^{\alpha} A p(t)+I_{t}^{\alpha} h(t) \\
& =\frac{t^{\alpha-1}}{\Gamma(\alpha)} x_{0}+I_{t}^{\alpha} A p(t)+I_{t}^{\alpha} h(t) .
\end{aligned}
$$

That is,

$$
p(t)=\frac{t^{\alpha-1}}{\Gamma(\alpha)} x_{0}+\frac{1}{\Gamma(\alpha)} \int_{0}^{t}(t-s)^{\alpha-1}[A p(s)+h(s)] d s
$$

Let $\lambda>0$; taking the Laplace transformations

$$
\widehat{p}(\lambda)=\int_{0}^{\infty} e^{-\lambda t} p(t) d t, \quad \widehat{h}(\lambda)=\int_{0}^{\infty} e^{-\lambda t} h(t) d t
$$

to (26), we obtain

$$
\begin{aligned}
\widehat{p}(\lambda) & =\frac{1}{\lambda^{\alpha}} x_{0}+\frac{1}{\lambda^{\alpha}} A \widehat{p}(\lambda)+\frac{1}{\lambda^{\alpha}} \widehat{h}(\lambda) \\
& =\left(\lambda^{\alpha} I-A\right)^{-1} x_{0}+\left(\lambda^{\alpha} I-A\right)^{-1} \widehat{h}(\lambda) \\
& =\int_{0}^{\infty} e^{-\lambda^{\alpha} t} T(t) x_{0} d t+\int_{0}^{\infty} e^{-\lambda^{\alpha} t} T(t) \widehat{h}(\lambda) d t
\end{aligned}
$$

Consider the one-sided stable probability density

$$
\begin{array}{r}
\omega_{\alpha}(\theta)=\frac{1}{\pi} \sum_{n=1}^{\infty}(-1)^{n-1} \theta^{-n \alpha-1} \frac{\Gamma(n \alpha+1)}{n !} \sin (n \pi \alpha), \\
\theta \in(0, \infty),
\end{array}
$$

whose Laplace transformation is given by

$$
\int_{0}^{\infty} e^{-\lambda \theta}{\omega_{\alpha}}(\theta) d \theta=e^{-\lambda^{\alpha}}, \quad \alpha \in(0,1)
$$

Hence, it follows from (28) and (30) that

$$
\begin{aligned}
& \int_{0}^{\infty} e^{-\lambda^{\alpha} t} T(t) x_{0} d t \\
& =\int_{0}^{\infty} e^{-(\lambda s)^{\alpha}} T\left(s^{\alpha}\right) x_{0} d s^{\alpha} \quad\left(t=s^{\alpha}\right) \\
& =\alpha \int_{0}^{\infty} e^{-(\lambda s)^{\alpha}} T\left(s^{\alpha}\right) s^{\alpha-1} x_{0} d s \\
& =\alpha \int_{0}^{\infty} \omega_{\alpha}(\theta) e^{-\lambda s \theta} T\left(s^{\alpha}\right) s^{\alpha-1} x_{0} d \theta d s \\
& =\alpha \iint_{0}^{\infty} \omega_{\alpha}(\theta) e^{-\lambda u} T\left(\frac{u^{\alpha}}{\theta^{\alpha}}\right) \frac{s^{\alpha-1}}{\theta^{\alpha}} x_{0} d u d \theta \quad(u=\theta s) \\
& =\int_{0}^{\infty} e^{-\lambda u}\left[\alpha \int_{0}^{\infty} \omega_{\alpha}(\theta) T\left(\frac{u^{\alpha}}{\theta^{\alpha}}\right) \frac{s^{\alpha-1}}{\theta^{\alpha}} x_{0} d \theta\right] d u, \\
& \int_{0}^{\infty} e^{-\lambda^{\alpha} t} T(t) \widehat{h}(\lambda) d t
\end{aligned}
$$

$$
\begin{aligned}
& =\int_{0}^{\infty} e^{-\lambda^{\alpha} t} T(t)\left[\int_{0}^{\infty} e^{-\lambda s} h(s) d s\right] d t \\
& =\iint_{0}^{\infty} e^{-\lambda^{\alpha} t} T(t) e^{-\lambda s} h(s) d s d t \\
& =\iint_{0}^{\infty} e^{-(\lambda \mu)^{\alpha}} T\left(\mu^{\alpha}\right) q \mu^{\alpha-1} e^{-\lambda s} h(s) d s d \mu \quad\left(t=\mu^{\alpha}\right) \\
& =\iiint_{0}^{\infty} \alpha \omega_{\alpha}(\theta) e^{-\lambda \mu \theta} T\left(\mu^{\alpha}\right) \mu^{\alpha-1} e^{-\lambda s} h(s) d \theta d s d \mu
\end{aligned}
$$$$
=\iiint_{0}^{\infty} \alpha \omega_{\alpha}(\theta) e^{-\lambda \nu} T\left(\frac{\nu^{\alpha}}{\theta^{\alpha}}\right)
$$$$
\times \frac{v^{\alpha-1}}{\theta^{\alpha}} e^{-\lambda s} h(s) d v d \theta d s \quad(\nu=\mu \theta)
$$$$
=\iiint_{0}^{\infty} \alpha{\omega_{\alpha}}(\theta) e^{-\lambda(\nu+s)} T\left(\frac{\nu^{\alpha}}{\theta^{\alpha}}\right) \frac{\nu^{\alpha-1}}{\theta^{\alpha}} h(s) d \nu d \theta d s
$$$$
=\iint_{0}^{\infty} \int_{s}^{\infty} \alpha \omega_{\alpha}(\theta) e^{-\lambda \tau} T\left(\frac{(\tau-s)^{\alpha}}{\theta^{\alpha}}\right)
$$$$
\times \frac{(\tau-s)^{\alpha-1}}{\theta^{\alpha}} h(s) d \tau d \theta d s \quad(\tau=\nu+s)
$$$$
=\int_{0}^{\infty} e^{-\lambda \tau}\left[\alpha \int_{0}^{\tau} \int_{0}^{\infty} \omega_{\alpha}(\theta) T\left(\frac{(\tau-s)^{\alpha}}{\theta^{\alpha}}\right)\right.
$$

$$
\left.\times \frac{(\tau-s)^{\alpha-1}}{\theta^{\alpha}} h(s) d \theta d s\right] d \tau .
$$


According to the above work, we get

$$
\begin{aligned}
\widehat{p}(\lambda)=\int_{0}^{\infty} e^{-\lambda t}\left[\alpha \int_{0}^{\infty} \omega_{\alpha}(\theta) T\left(\frac{t^{\alpha}}{\theta^{\alpha}}\right) \frac{t^{\alpha-1}}{\theta^{\alpha}} x_{0} d \theta\right. \\
+\alpha \int_{0}^{t} \int_{0}^{\infty} \omega_{\alpha}(\theta) T\left(\frac{(t-s)^{\alpha}}{\theta^{\alpha}}\right) \\
\left.\times \frac{(t-s)^{\alpha-1}}{\theta^{\alpha}} h(s) d \theta d s\right] d t .
\end{aligned}
$$

Now, we can invert the Laplace transform to (20) and obtain

$$
\begin{aligned}
p(t)= & \alpha \int_{0}^{\infty} \omega_{\alpha}(\theta) T\left(\frac{t^{\alpha}}{\theta^{\alpha}}\right) \frac{t^{\alpha-1}}{\theta^{\alpha}} x_{0} d \theta \\
& +\alpha \int_{0}^{t} \int_{0}^{\infty} \omega_{\alpha}(\theta) T\left(\frac{(t-s)^{\alpha}}{\theta^{\alpha}}\right) \frac{(t-s)^{\alpha-1}}{\theta^{\alpha}} h(s) d \theta d t \\
= & \alpha \int_{0}^{\infty} \frac{1}{\alpha} \theta^{-1-(1 / \alpha)} \omega_{\alpha}\left(\theta^{-1 / \alpha}\right) \theta T\left(t^{\alpha} \theta\right) t^{\alpha-1} x_{0} d \theta \\
& +\alpha \int_{0}^{t} \int_{0}^{\infty}(t-s)^{\alpha-1} \frac{1}{\alpha} \theta^{-1-(1 / \alpha)} \omega_{\alpha}\left(\theta^{-1 / \alpha}\right) \theta T \\
& \times\left((t-s)^{\alpha} \theta\right) h(s) d \theta d t .
\end{aligned}
$$

Let

$$
\begin{gathered}
\xi_{\alpha}(\theta)=\frac{1}{\alpha} \theta^{-1-(1 / \alpha)} \omega_{\alpha}\left(\theta^{-1 / \alpha}\right), \\
T_{\alpha}(t)=\alpha \int_{0}^{\infty} \theta \xi_{\alpha}(\theta) T\left(t^{\alpha} \theta\right) d \theta .
\end{gathered}
$$

Then, we get

$p(t)=t^{\alpha-1} T_{\alpha}(t) x_{0}+\int_{0}^{t}(t-s)^{\alpha-1} T_{\alpha}(t-s) h(s) d s$.

Now we calculate the $P C_{1-\alpha}$-mild solution of (24).

Applying Riemann-Liouville fractional integral operator on both sides of (24), then by Lemma 4 , we get

$q(t)$

$$
\begin{aligned}
& \int \frac{1}{\Gamma(\alpha)} \int_{0}^{t}(t-s)^{\alpha-1} A q(s) d s, \quad t \in\left[0, t_{1}\right], \\
& =\left\{\sum_{k=1}^{m} \frac{I_{k}\left(x\left(t_{k}^{-}\right)\right)}{\Gamma(\alpha)}\left(t-t_{i}\right)^{\alpha-1}\right. \\
& +\frac{1}{\Gamma(\alpha)} \int_{0}^{t}(t-s)^{\alpha-1} A q(s) d s, \quad t \in\left(t_{k}, t_{k+1}\right] .
\end{aligned}
$$

where

$$
\chi_{k}(t)= \begin{cases}0, & t \leq t_{k} \\ 1, & t>t_{k}\end{cases}
$$

Let $\lambda>0$; taking the Laplace transformation to (37), we obtain

$$
\widehat{q}(\lambda)=\sum_{k=1}^{m} \frac{I_{k}\left(x\left(t_{k}^{-}\right)\right) e^{-\lambda t_{k}}}{\lambda^{\alpha}}+\frac{1}{\lambda^{\alpha}} A \widehat{q}(\lambda) .
$$

That is,

$$
\widehat{q}(\lambda)=\sum_{k=1}^{m}\left(\lambda^{\alpha} I-A\right)^{-1} I_{k}\left(x\left(t_{k}^{-}\right)\right) e^{-\lambda t_{k}} .
$$

Notice that the Laplace transform of $t^{\alpha-1} T_{\alpha}(t) y_{k}$ is $\left(\lambda^{\alpha} I-\right.$ $A)^{-1} y_{k}$. Thus one can calculate the mild solution of (24) as

$$
q(t)=\sum_{k=1}^{m} \chi_{k}(t)\left(t-t_{i}\right)^{\alpha-1} T_{\alpha}\left(t-t_{i}\right) I_{k}\left(x\left(t_{k}^{-}\right)\right) .
$$

By the above work, the $P C_{1-\alpha}$-mild solution of (19) is given by

$$
\begin{aligned}
x(t)= & t^{\alpha-1} T_{\alpha}(t) x_{0} \\
& +\sum_{k=1}^{m} \chi_{k}(t)\left(t-t_{k}\right)^{\alpha-1} T_{\alpha}\left(t-t_{k}\right) I_{k}\left(x\left(t_{k}^{-}\right)\right) \\
& +\int_{0}^{t}(t-s)^{\alpha-1} T_{\alpha}(t-s) h(s) d s .
\end{aligned}
$$

That is,

$$
\begin{aligned}
& x(t) \\
& \left\{\begin{array}{c}
t^{\alpha-1} T_{\alpha}(t) x_{0}+\int_{0}^{t}(t-s)^{\alpha-1} T_{\alpha}(t-s) h(s) d s \\
t \in\left[0, t_{1}\right] \\
t^{\alpha-1} T_{\alpha}(t) x_{0}+\sum_{k=1}^{m} T_{\alpha}\left(t-t_{k}\right)\left(t-t_{k}\right)^{\alpha-1} I_{k}\left(x\left(t_{k}^{-}\right)\right) \\
+\int_{0}^{t}(t-s)^{\alpha-1} T_{\alpha}(t-s) h(s) d s, \\
t \in\left(t_{k}, t_{k+1}\right]
\end{array}\right.
\end{aligned}
$$

where $k=1,2, \ldots, m$,

$$
\begin{gathered}
T_{\alpha}(t)=\alpha \int_{0}^{\infty} \theta \xi_{\alpha}(\theta) T\left(t^{\alpha} \theta\right) d \theta, \\
\xi_{\alpha}(\theta)=\frac{1}{\alpha} \theta^{-1-(1 / \alpha)} ळ_{\alpha}\left(\theta^{-1 / \alpha}\right), \\
\omega_{\alpha}(\theta)=\frac{1}{\pi} \sum_{n=1}^{\infty}(-1)^{n-1} \theta^{-n \alpha-1} \frac{\Gamma(n \alpha+1)}{n !} \sin (n \pi \alpha),
\end{gathered}
$$


where $\xi_{\alpha}$ is a probability density function defined on $(0, \infty)$; that is,

$$
\xi_{\alpha}(\theta) \geq 0, \quad \theta \in(0, \infty), \quad \int_{0}^{\infty} \xi_{\alpha}(\theta) d \theta=1 .
$$

This completes the proof of the lemma.

According to Lemma 5, we give the following definition.

Definition 6. A function $x \in P C_{1-\alpha}(J, X)$ is called a mild solution of (4) if $\left.I_{t}^{1-\alpha} x(t)\right|_{t=0}=x_{0}$ and there exits $f \in L^{1}(J<$ $X)$ such that $f(t) \in F(t, x(t))$ a.e. on $t \in J$ and

$$
\begin{aligned}
& x(t) \\
& =\left\{\begin{array}{r}
t^{\alpha-1} T_{\alpha}(t) x_{0} \\
+\int_{0}^{t}(t-s)^{\alpha-1} T_{\alpha}(t-s)[B u(s)+f(s, x(s))] d s, \\
t \in\left[0, t_{1}\right], \\
t^{\alpha-1} T_{\alpha}(t) x_{0}+\sum_{k=1}^{m} T_{\alpha}\left(t-t_{k}\right)\left(t-t_{k}\right)^{\alpha-1} I_{k}\left(x\left(t_{k}^{-}\right)\right) \\
+\int_{0}^{t}(t-s)^{\alpha-1} T_{\alpha}(t-s)[B u(s)+f(s, x(s))] d s, \\
t \in\left(t_{k}, t_{k+1}\right],
\end{array}\right.
\end{aligned}
$$

where $k=1,2, \ldots, m$,

$$
\begin{gathered}
T_{\alpha}(t)=\alpha \int_{0}^{\infty} \theta \xi_{\alpha}(\theta) T\left(t^{\alpha} \theta\right) d \theta, \\
\xi_{\alpha}(\theta)=\frac{1}{\alpha} \theta^{-1-(1 / \alpha)} \varrho_{\alpha}\left(\theta^{-1 / \alpha}\right), \\
\omega_{\alpha}(\theta)=\frac{1}{\pi} \sum_{n=1}^{\infty}(-1)^{n-1} \theta^{-n \alpha-1} \frac{\Gamma(n \alpha+1)}{n !} \sin (n \pi \alpha), \\
\theta \in(0, \infty),
\end{gathered}
$$

where $\xi_{\alpha}$ is a probability density function defined on $(0, \infty)$; that is,

$$
\xi_{\alpha}(\theta) \geq 0, \quad \theta \in(0, \infty), \quad \int_{0}^{\infty} \xi_{\alpha}(\theta) d \theta=1 .
$$

Due to the work of the paper [31], we have the following result.

Lemma 7. The operator $T_{\alpha}(t)$ has the following properties.

(i) For any fixed $t \geq 0, T_{\alpha}(t)$ is linear and bounded operator; that is, for any $x \in X$,

$$
\left\|T_{\alpha}(t) x\right\| \leq \frac{M}{\Gamma(\alpha)}\|x\| .
$$

(ii) $T_{\alpha}(t)(t \geq 0)$ is strongly continuous.

Now, we also introduce some basic definitions on multivalued maps. For more details, see [36-38].
A multivalued map $G: X \rightarrow \mathscr{P}(X)$ is convex (closed) valued if $G(x)$ is convex (closed) for all $x \in X$. $G$ is bounded on bounded sets if $G(B)=\bigcup_{x \in B} . G(x)$ is bounded on $X$ for any bounded set $B$ of $X$; that is, $\sup _{x \in B}\{\sup \{\|y\|: y \in$ $G(x)\}\}<\infty$.

$G$ is called upper semicontinuous (u.s.c.) on $X$ if, for each $x_{0} \in X$, the set $G\left(x_{0}\right)$ is a nonempty closed subset of $X$, and if, for each open set $U$ of $X$ containing $G\left(x_{0}\right)$, there exists an open neighborhood $V$ of $x_{0}$ such that $G(V) \subseteq U$.

$G$ is said to be completely continuous if $G(B)$ is relatively compact for every $B \in P_{b}(X)$.

If the multivalued map $G$ is completely continuous with nonempty compact values, then $G$ is u.s.c. if and only if $G$ has a closed graph (i.e., $x_{n} \rightarrow x_{*}, y_{n} \rightarrow y_{*}, y_{n} \in G\left(x_{n}\right)$ imply $\left.y_{*} \in G\left(x_{*}\right)\right)$.

We say that $G$ has a fixed point if there is a $x \in X$ such that $x \in G(x)$.

A multivalued map $G: J \rightarrow P_{c l}(X)$ is said to be measurable if for each $x \in X$ the function $Y: J \rightarrow R^{+}$ defined by $Y(t)=d(x, G(t))=\inf \{\|x-z\|: z \in G(t)\}$ is measurable.

Definition 8 . The system (4) is said to be exactly controllable on $J$, if, for all $x_{0}, x_{1} \in X$, there exists a control $u \in L^{p}(J, U)$ $(p>1 / \alpha)$ such that the mild solution of $(4)$ satisfies $x(0 ; u)=$ $x_{0}$ and $x(b ; u)=x_{1}$.

Definition 9. The system (4) is said to be approximately controllable on the interval $J$, if, for all $x_{0} \in X$, one has $\overline{\mathscr{R}\left(b, x_{0}\right)}=X$, where $\overline{\mathscr{R}\left(b, x_{0}\right)}=\left\{x(b ; u): u \in L^{p}(J, U)(p>\right.$ $\left.1 / \alpha), x(0 ; u)=x_{0}\right\}$ is the reachable set of system (4) with the initial values $x_{0}, x_{1}$ at the terminal time $b$.

It is convenient at this point to introduce two relevant operators:

$$
\begin{gathered}
\Gamma_{0}^{b}=\int_{0}^{b}(b-s)^{2 \alpha-2} T_{\alpha}(b-s) B B^{*} T_{\alpha}^{*}(b-s) d s, \\
\frac{1}{2}<\alpha \leq 1, \\
R\left(a, \Gamma_{0}^{b}\right)=\left(a I+\Gamma_{0}^{b}\right)^{-1}, \quad a>0,
\end{gathered}
$$

where $B^{*}$ denotes the adjoint of $B$ and $T_{\alpha}^{*}(t)$ is the adjoint of $T_{\alpha}(t)$. It is straightforward that the operator $\Gamma_{0}^{b}$ is a linear bounded operator.

We consider the following linear fractional differential system:

$$
\begin{gathered}
{ }^{c} D^{\alpha} x(t)=A x(t)+B u(t), \quad \frac{1}{2}<\alpha \leq 1, t \in J=(0, b], \\
\left.I_{t}^{1-\alpha} x(t)\right|_{t=0}=x_{0} \in X .
\end{gathered}
$$

Lemma 10. The linear fractional differential system (51) is approximately controllable on $J$ if and only if $a R\left(a, \Gamma_{0}^{b}\right) \rightarrow 0$ as $a \rightarrow 0^{+}$in the strong operator topology.

The proof of this lemma is a straightforward adaptation of the proof of [3]. 
Lemma 11. Let $E$ be a Banach space and let $\mathscr{W} \subset L^{1}(J, E)$ be integrably bounded. If, for all $t \in J$, there is a relatively weakly compact set $C(t) \subset E$ such that $w(t) \in C(t)$ for every $w \in \mathscr{W}$, then $\mathscr{W}$ is relatively weakly compact in $L^{1}(J, E)$.

Lemma 12 (Lasota and Opial [39]). Let $J$ be a compact real interval and let $X$ be a Banach space. The multivalued map $F: J \times X \rightarrow \mathscr{P}_{b, c l, c v}(X)$ is measurable to $t$ for each fixed $x \in X$, u.s.c. to $x$ for each $t \in J$, and for each $x \in C(J, X)$ the set $S_{F, x}=\left\{f \in L^{1}(J, X): f(t) \in F(t, x(t)), t \in J\right\}$ is nonempty. Let $\Gamma$ be a linear continuous mapping from $L^{1}(J, X)$ to $C(J, X)$; then, the operator

$$
\begin{aligned}
\Gamma \circ S_{F} & : C(J, X) \longrightarrow \mathscr{P}_{b, c l, c v}(C(J, X)), \\
x & \longmapsto\left(\Gamma \circ S_{F}\right)(x)=\Gamma\left(S_{F, x}\right)
\end{aligned}
$$

is a closed graph operator in $C(J, X) \times C(J, X)$.

Lemma 13 (see [37]). Let $D$ be a bounded, convex, and closed subset in the Banach space $E$ and let $G: D \rightarrow 2^{X} \backslash\{\emptyset\}$ be a u.s.c. condensing multivalued map. If, for every $x \in D, G(x)$ is a closed and convex set in $D$, then $G$ has a fixed point.

\section{Main Results}

In this section, we present our main result on approximate controllability of system (4). To do this, we first prove the existence of solutions for fractional control system. Secondly, we show that, under certain assumptions, the approximate controllability of (4) is implied by the approximate controllability of the corresponding linear system.

For convenience, let us introduce some notations:

$$
\begin{gathered}
M_{b}=\|B\|, \quad \beta=\left(\frac{1-\gamma}{\alpha-\gamma} b^{(\alpha-\gamma) /(1-\gamma)}\right)^{1-\gamma}, \\
\Lambda=\frac{M \beta}{\Gamma(\alpha)}+\frac{M^{3} M_{B}^{2} b^{2 \alpha-1} \beta}{a(2 \alpha-1)(\Gamma(\alpha))^{3}} .
\end{gathered}
$$

Before stating and proving our main results, we introduce the following assumptions.

$H(1): T_{\alpha}(t)$ is compact, $\left\|a R\left(a, \Gamma_{0}^{b}\right)\right\| \leq 1$.

$H(2): F$ is a multivalued map satisfying $F: J \times$ $X \rightarrow \mathscr{P}_{c p, c v}(X)$ which is measurable to $t$ for each fixed $x \in D$, u.s.c. to $x$ for each $t \in J$, and for each $x \in P C_{1-\alpha}(J, X)$ the set

$$
S_{F, x}=\left\{f \in L^{1}(J, X): f(t) \in F(t, x(t))\right\}
$$

is nonempty.

$H(3)$ : There exist a function $P(t) \in L^{1 / \gamma}\left(J, R^{+}\right), \gamma \in$ $(0, \alpha)$, and a nondecreasing continuous function $\psi$ : $\rightarrow R^{+}$, such that

$$
\begin{aligned}
\|F(t, x(t))\|_{X} & =\sup \left\{\|f(t)\|_{X}: f(t) \in F(t, x(t))\right\} \\
& \leq P(t) \psi\left(\|x\|_{D}\right),
\end{aligned}
$$

for a.e. $t \in J$, for all $x \in D$, and for each $r>0$, there exists $0<\rho<1$, such that

$$
\lim _{r \rightarrow \infty} \inf \frac{\psi(r)}{r}\|P\|_{L^{2}}=\rho<1 .
$$

$H(4)$ : There exist constants $d_{k}>0, k=1,2, \ldots, m$, with $(M / \Gamma(\alpha)) \sum_{k=1}^{m} d_{k}<1$ such that

$$
\left\|I_{k}(x)-I_{k}(y)\right\| \leq d_{k}\left(t-t_{k}\right)^{1-\alpha}\|x-y\|_{X}, \quad \forall x, y \in X .
$$

Theorem 14. If the conditions $H(1)-H(4)$ are held, then the system (4) has a mild solution.

Proof. We consider a set

$$
B_{r}=\left\{x \in P C_{1-\alpha}(J, X):\|x\| \leq r, r>0\right\}
$$

on the space $P C_{1-\alpha}(J, X)$. We easily know that $B_{r}$ is a bounded, closed, and convex set in $P C_{1-\alpha}(J, X)$. For $a>0$, for all $x(\cdot) \in P C_{1-\alpha}(J, X), x_{1} \in X$, we take the control function as

$$
u(t)=(b-t)^{\alpha-1} B^{*} T_{\alpha}^{*}(b-t) R\left(a, \Gamma_{0}^{b}\right) P(x(\cdot)),
$$

where

$$
\begin{aligned}
P(x(\cdot))= & x_{1}-t^{\alpha-1} T_{\alpha}(b) x_{0} \\
& -\sum_{k=1}^{m} T_{\alpha}\left(t-t_{k}\right)\left(t-t_{k}\right)^{\alpha-1} I_{k}\left(x\left(t_{k}^{-}\right)\right) \\
& -\int_{0}^{b}(b-s)^{\alpha-1} T_{\alpha}(b-s) f(s) d s, \quad f \in S_{F, x} .
\end{aligned}
$$

By this control, we define the operator $\Phi_{a}: P C_{1-\alpha}(J, X) \rightarrow$ $\mathscr{P}\left(P C_{1-\alpha}(J, X)\right)$ as follows:

$$
\begin{aligned}
\Phi_{a}(x)=\{ & \tau \in P C_{1-\alpha}(J, X) \\
& : \tau(t)=t^{\alpha-1} T_{\alpha}(t) x_{0} \\
& +\int_{0}^{t}(t-s)^{\alpha-1} T_{\alpha}(t-s) f(s) d s \\
& +\int_{0}^{t}(t-s)^{\alpha-1} T_{\alpha}(t-s) B u(s) d s, \\
& f \in S_{F, x}, t \in\left(0, t_{1}\right], \\
& t^{\alpha-1} T_{\alpha}(t) x_{0} \\
& +\sum_{k=1}^{m} T_{\alpha}\left(t-t_{k}\right)\left(t-t_{k}\right)^{\alpha-1} I_{k}\left(x\left(t_{k}^{-}\right)\right)
\end{aligned}
$$




$$
\begin{aligned}
& +\int_{0}^{t}(t-s)^{\alpha-1} T_{\alpha}(t-s) f(s) d s \\
& +\int_{0}^{t}(t-s)^{\alpha-1} \\
& \quad \times T_{\alpha}(t-s) B u(s) d s, \\
& \left.f \in S_{F, x}, t \in\left(t_{k}, t_{k+1}\right], k=1,2, \ldots, m .\right\} .
\end{aligned}
$$

We will show that, for all $a>0$, the operator $\Phi_{a}$ : $P C_{1-\alpha}(J, X) \rightarrow \mathscr{P}\left(P C_{1-\alpha}(J, X)\right)$ has a fixed point. For the sake of convenience, we subdivide the proof into several steps.

Step 1. For each $a>0$, the operator $\Phi_{a}(x)$ is convex for each $x \in P C_{1-\alpha}(J, X)$.

In fact, if $\tau_{1}, \tau_{2} \in \Phi_{a}(x)$, then, for each $t \in\left(t_{k}, t_{k+1}\right], k=$ $1,2, \ldots, m$, there exist $f_{1}, f_{2} \in S_{F, x}$ such that

$$
\begin{aligned}
\tau_{i}(t)= & t^{\alpha-1} T_{\alpha}(t) x_{0} \\
& +\sum_{k=1}^{m} T_{\alpha}\left(t-t_{k}\right)\left(t-t_{k}\right)^{\alpha-1} I_{k}\left(x\left(t_{k}^{-}\right)\right) \\
& +\int_{0}^{t}(t-s)^{\alpha-1} T_{\alpha}(t-s) f_{i}(s) d s \\
& +\int_{0}^{t}(t-s)^{\alpha-1} T_{\alpha}(t-s) B B^{*} T_{\alpha}^{*}(b-t) R\left(a, \Gamma_{0}^{b}\right) \\
& \times\left[x_{1}-t^{\alpha-1} T_{\alpha}(t) x_{0}\right. \\
& -\sum_{k=1}^{m} T_{\alpha}\left(t-t_{k}\right)\left(t-t_{k}\right)^{\alpha-1} I_{k}\left(x\left(t_{k}^{-}\right)\right) \\
& \left.\quad-\int_{0}^{b}(b-\mu)^{\alpha-1} T_{\alpha}(b-\mu) f_{i}(\mu) d \mu\right] d s \\
& i=1,2 .
\end{aligned}
$$

Let $0 \leq \lambda \leq 1$; then, for each $t \in\left(t_{k}, t_{k+1}\right], k=1,2, \ldots, m$, we have

$$
\begin{aligned}
& \lambda \tau_{1}(t)+(1-\lambda) \tau_{2}(t) \\
& =t^{\alpha-1} T_{\alpha}(t) x_{0}+\sum_{k=1}^{m} T_{\alpha}\left(t-t_{k}\right)\left(t-t_{k}\right)^{\alpha-1} I_{k}\left(x\left(t_{k}^{-}\right)\right) \\
& \quad+\int_{0}^{t}(t-s)^{\alpha-1} T_{\alpha}(t-s)\left[\lambda f_{1}(s)+(1-\lambda) f_{2}(s)\right] d s
\end{aligned}
$$

$$
\begin{gathered}
+\int_{0}^{t}(t-s)^{\alpha-1} T_{\alpha}(t-s) B B^{*} T_{\alpha}^{*}(b-t) R\left(a, \Gamma_{0}^{b}\right) \\
\times\left[x_{1}-t^{\alpha-1} T_{\alpha}(t) x_{0}\right. \\
\quad-\sum_{k=1}^{m} T_{\alpha}\left(t-t_{k}\right)\left(t-t_{k}\right)^{\alpha-1} I_{k}\left(x\left(t_{k}^{-}\right)\right) \\
\quad-\int_{0}^{b}(b-\mu)^{\alpha-1} T_{\alpha}(b-\mu) \\
\left.\quad \times\left[\lambda f_{1}(\mu)+(1-\lambda) f_{2}(\mu)\right] d \mu\right] d s .
\end{gathered}
$$

Since $S_{F, x}$ is convex (because $F$ has convex values), $\lambda f_{1}+(1-$ d) $f_{2} \in S_{F, x}$; thus, $\lambda \tau_{1}(t)+(1-\lambda) \tau_{2} \in \Phi_{a}(x)$.

Step 2. For each $a>0$, there is a positive constant $r_{0}=r(a)$, such that $\Phi_{a}\left(B_{r_{0}}\right) \subset B_{r_{0}}$.

If this is not true, then there exists $a>0$ such that, for every $r>0$, there exists a $\bar{x} \in B_{r}$ such that $\Phi_{a}(\bar{x}) \nsubseteq B_{r}$; that is,

$$
\begin{gathered}
\left\|\Phi_{a}(\bar{x})\right\| \equiv \sup \left\{\|\tau\|_{P C_{1-\alpha}(J, X)}: \tau \in \Phi_{a}(\bar{x})\right\}>r, \\
\tau(t)=t^{\alpha-1} T_{\alpha}(t) x_{0} \\
+\sum_{k=1}^{m} T_{\alpha}\left(t-t_{k}\right)\left(t-t_{k}\right)^{\alpha-1} I_{k}\left(x\left(t_{k}^{-}\right)\right) \\
+\int_{0}^{t}(t-s)^{\alpha-1} T_{\alpha}(t-s) f(s) d s \\
+\int_{0}^{t}(t-s)^{\alpha-1} T_{\alpha}(t-s) B B^{*} T_{\alpha}^{*}(b-t) R\left(a, \Gamma_{0}^{b}\right) \\
\times\left[x_{1}-b^{\alpha-1} T_{\alpha}(b) x_{0}\right. \\
\quad-\sum_{k=1}^{m} T_{\alpha}\left(b-t_{k}\right)\left(b-t_{k}\right)^{\alpha-1} I_{k}\left(x\left(t_{k}^{-}\right)\right) \\
\left.\quad-\int_{0}^{b}(b-\mu)^{\alpha-1} T_{\alpha}(b-\mu) f(\mu) d \mu\right] d s,
\end{gathered}
$$

for some $S_{F, \bar{x}}$.

By using Holder's inequality and $H(3)$, we have

$$
\begin{gathered}
\int_{0}^{t}(t-s)^{\alpha-1}\|u(s)\| d s \\
\leq \frac{M M_{B} b^{2 \alpha-1}}{a \Gamma(\alpha)(2 \alpha-1)}
\end{gathered}
$$


10

Abstract and Applied Analysis

$$
\begin{aligned}
& \times\left[\left\|x_{1}\right\|+\frac{M\left\|x_{0}\right\|}{\Gamma(\alpha)}\right. \\
& +\sum_{k=1}^{m} \frac{M}{\Gamma(\alpha)}\left(d_{k}\|x\|+m\left(b-t_{k}\right)^{\alpha-1}\left\|I_{k}(0)\right\|\right) \\
& \left.\quad+\frac{M \psi(r) \beta}{\Gamma(\alpha)}\|P\|_{L^{1 / \gamma}}\right] .
\end{aligned}
$$

Then, we obtain

$$
\begin{aligned}
& \left(t-t_{k}\right)^{1-\alpha}\|\tau(t)\| \\
& \leq\left(t-t_{k}\right)^{1-\alpha}\left\|t^{\alpha-1} T_{\alpha}(t) x_{0}\right\|+\left(t-t_{k}\right)^{1-\alpha} \\
& \times \sum_{k=1}^{m}\left\|T_{\alpha}\left(t-t_{k}\right)\right\|\left(t-t_{k}\right)^{\alpha-1}\left\|I_{k}\left(x\left(t_{k}^{-}\right)\right)\right\| \\
& +\left(t-t_{k}\right)^{1-\alpha} \\
& \times \int_{0}^{t}(t-s)^{\alpha-1}\left\|T_{\alpha}(t-s)\right\|\|f(s)\| d s \\
& +\left(t-t_{k}\right)^{1-\alpha} \\
& \times \int_{0}^{t}(t-s)^{\alpha-1}\left\|T_{\alpha}(t-s)\right\|\|B\|\|u(s)\| d s \\
& \leq \frac{M b^{\alpha-1}\left(b-t_{k}\right)^{1-\alpha}}{\Gamma(\alpha)}\left\|x_{0}\right\|+\left(b-t_{k}\right)^{1-\alpha} \\
& \times \sum_{k=1}^{m} \frac{M}{\Gamma(\alpha)}\left(d_{k}\|x\|+m\left(b-t_{k}\right)^{\alpha-1}\left\|I_{k}(0)\right\|\right) \\
& +\frac{M\left(b-t_{k}\right)^{1-\alpha}}{\Gamma(\alpha)} \int_{0}^{t}(t-s)^{\alpha-1} P(s) \psi(r) d s \\
& +\frac{M M_{B}\left(b-t_{k}\right)^{1-\alpha}}{\Gamma(\alpha)} \int_{0}^{t}(t-s)^{\alpha-1} u(s) d s \\
& \leq \frac{M b^{\alpha-1}\left(b-t_{k}\right)^{1-\alpha}}{\Gamma(\alpha)}\left\|x_{0}\right\|+\left(b-t_{k}\right)^{1-\alpha} \\
& \times \sum_{k=1}^{m} \frac{M}{\Gamma(\alpha)}\left(d_{k}\|x\|+m\left(b-t_{k}\right)^{\alpha-1}\left\|I_{k}(0)\right\|\right) \\
& +\frac{b^{2 \alpha-1} M^{2} M_{B}^{2}\left(b-t_{k}\right)^{1-\alpha}}{a(2 \alpha-1)(\Gamma(\alpha))^{2}} \\
& \times\left[\left\|x_{1}\right\|+\frac{M\left\|x_{0}\right\|}{\Gamma(\alpha)}\right. \\
& \left.+\sum_{k=1}^{m} \frac{M}{\Gamma(\alpha)}\left(d_{k}\|x\|+m\left(b-t_{k}\right)^{\alpha-1}\left\|I_{k}(0)\right\|\right)\right] \\
& +\psi(r) \Lambda\|P\|_{L^{1 / \gamma}} .
\end{aligned}
$$

Thus,

$$
\begin{aligned}
r \leq & \frac{M b^{\alpha-1}\left(b-t_{k}\right)^{1-\alpha}}{\Gamma(\alpha)}\left\|x_{0}\right\|+\left(b-t_{k}\right)^{1-\alpha} \\
& \times \sum_{k=1}^{m} \frac{M}{\Gamma(\alpha)}\left(d_{k}\|x\|\right. \\
& \left.+m\left(b-t_{k}\right)^{\alpha-1}\left\|I_{k}(0)\right\|\right) \\
+ & \frac{b^{2 \alpha-1} M^{2} M_{B}^{2}\left(b-t_{k}\right)^{1-\alpha}}{a(2 \alpha-1)(\Gamma(\alpha))^{2}} \\
& \times\left[\left\|x_{1}\right\|+\frac{M\left\|x_{0}\right\|}{\Gamma(\alpha)}\right. \\
& \left.+\sum_{k=1}^{m} \frac{M}{\Gamma(\alpha)}\left(d_{k}\|x\|+m\left(b-t_{k}\right)^{\alpha-1}\left\|I_{k}(0)\right\|\right)\right] \\
+ & +(r) \Lambda\|P\|_{L^{1 / \gamma}} .
\end{aligned}
$$

Dividing both sides by $r$ and taking the low limit as $r \rightarrow \infty$, we get

$$
1 \leq \lim _{r \rightarrow \infty} \inf \frac{\psi(r)}{r}\|P\|_{L^{1 / \gamma}}
$$

which is a contradiction to $H(3)$. Thus, for each $a>0$, there exists $r_{0}$ such that $\Phi_{a}(x)$ maps $B_{r_{0}}$ into itself.

Step 3. $\Phi_{\alpha}(x)$ is closed for each $x \in P C_{1-\alpha}(J, X)$.

Indeed, for each given $x \in P C_{1-\alpha}(J, X)$, let $\left\{\tau_{n}\right\}_{n \geq 0} \subset$ $\Phi_{a}(x)$ such that $\tau_{n} \rightarrow \tau$ in $P C_{1-\alpha}(J, X)$. Then, there exists $f_{n} \in S_{F, x}$ such that, for each $t \in J$,

$$
\begin{aligned}
\tau_{n}(t)= & t^{\alpha-1} T_{\alpha}(t) x_{0} \\
& +\sum_{k=1}^{m} T_{\alpha}\left(t-t_{k}\right)\left(t-t_{k}\right)^{\alpha-1} I_{k}\left(x\left(t_{k}\right)\right) \\
& +\int_{0}^{t}(t-s)^{\alpha-1} T_{\alpha}(t-s) f_{n}(s) d s \\
& +\int_{0}^{t}(t-s)^{\alpha-1} T_{\alpha}(t-s) B u_{n}(s) d s,
\end{aligned}
$$

where

$$
\begin{aligned}
u_{n}(t)= & B^{*} T_{\alpha}^{*}(b-t) R\left(a, \Gamma_{0}^{b}\right) \\
& \times\left[x_{1}-b^{\alpha-1} T_{\alpha}(b) x_{0}\right. \\
& \quad-\sum_{k=1}^{m} T_{\alpha}\left(b-t_{k}\right)\left(b-t_{k}\right)^{\alpha-1} I_{k}\left(x\left(t_{k}^{-}\right)\right) \\
& \left.\quad-\int_{0}^{b}(b-s)^{\alpha-1} T_{\alpha}(b-s) f_{n}(s) d s\right] .
\end{aligned}
$$


Because of [40, Proposition 3.1], $S_{F, x}$ is weakly compact in $L^{1}(J, X)$ which implies that $f_{n}$ converges weakly to some $f \in$ $S_{F, x}$ in $L^{1}(J, X)$. Thus, $u_{n} \rightarrow u$, and

$$
\begin{aligned}
u(t)=B^{*} T_{\alpha}^{*}(b-t) R\left(a, \Gamma_{0}^{b}\right) & \\
\times & {\left[x_{1}-b^{\alpha-1} T_{\alpha}(b) x_{0}\right.} \\
& \quad-\sum_{k=1}^{m} T_{\alpha}\left(b-t_{k}\right)\left(b-t_{k}\right)^{\alpha-1} I_{k}\left(x\left(t_{k}^{-}\right)\right) \\
& \left.\quad-\int_{0}^{b}(b-s)^{\alpha-1} T_{\alpha}(b-s) f(s) d s\right] .
\end{aligned}
$$

Then, for each $t \in J$,

$$
\begin{aligned}
& \tau_{n}(t) \longrightarrow \tau(t) \\
& =t^{\alpha-1} T_{\alpha}(t) x_{0} \\
& +\sum_{k=1}^{m} T_{\alpha}\left(t-t_{k}\right)\left(t-t_{k}\right)^{\alpha-1} I_{k}\left(x\left(t_{k}^{-}\right)\right) \\
& +\int_{0}^{t}(t-s)^{\alpha-1} T_{\alpha}(t-s) f(s) d s \\
& +\int_{0}^{t}(t-s)^{\alpha-1} T_{\alpha}(t-s) B B^{*} T_{\alpha}^{*}(b-t) R\left(a, \Gamma_{0}^{b}\right) \\
& \times\left[x_{1}-b^{\alpha-1} T_{\alpha}(b) x_{0}\right. \\
& -\sum_{k=1}^{m} T_{\alpha}\left(b-t_{k}\right)\left(b-t_{k}\right)^{\alpha-1} I_{k}\left(x\left(t_{k}^{-}\right)\right) \\
& \left.-\int_{0}^{b}(b-\mu)^{\alpha-1} T_{\alpha}(b-\mu) f(\mu) d \mu\right] d s .
\end{aligned}
$$

Thus, we show that $\tau \in \Phi_{a}(x)$.

Step 4. $\Phi_{a}$ is u.s.c and condensing.

We decompose $\Phi_{a}$ as $\Phi_{a}=\Phi_{a}^{1}+\Phi_{a}^{2}$, where the operators $\Phi_{a}^{1}$ and $\Phi_{a}^{2}$ are defined by

$$
\begin{aligned}
& \left(\Phi_{a}^{1} x\right)(t)=\sum_{k=1}^{m} T_{\alpha}\left(t-t_{k}\right)\left(t-t_{k}\right)^{\alpha-1} I_{k}\left(x\left(t_{k}^{-}\right)\right), \\
& t \in J, k=1,2, \ldots, m, \\
& \Phi_{a}^{2}(x)=\left\{\tau \in P C_{1-\alpha}(J, X): \tau(t)\right. \\
& =t^{\alpha-1} T_{\alpha}(t) x_{0} \\
& +\int_{0}^{t}(t-s)^{\alpha-1} T_{\alpha}(t-s) f(s) d s
\end{aligned}
$$

$$
\begin{aligned}
& +\int_{0}^{t}(t-s)^{\alpha-1} T_{\alpha}(t-s) \\
& \times B B^{*} T_{\alpha}^{*}(b-t) R\left(a, \Gamma_{0}^{b}\right) \\
& \times\left[x_{1}-b^{\alpha-1} T_{\alpha}(b) x_{0}\right. \\
& \quad-\sum_{k=1}^{m} T_{\alpha}\left(b-t_{k}\right)\left(b-t_{k}\right)^{\alpha-1} I_{k}\left(x\left(t_{k}^{-}\right)\right) \\
& \left.\quad-\int_{0}^{b}(b-\mu)^{\alpha-1} T_{\alpha}(b-\mu) f(\mu) d \mu\right] d s, \\
& \left.f \in S_{F, x}, t \in J\right\} .
\end{aligned}
$$

According to [41, Corollary 2.2.1], we will prove that $\Phi_{a}^{1}$ is a contraction operator, while $\Phi_{a}^{2}$ is a completely continuous operator.

Let us begin proving that $\Phi_{a}^{1}$ is a contraction operator. For any $x, y \in X$, we obtain

$$
\begin{aligned}
& \left\|\left(\Phi_{a}^{1} x\right)(t)-\left(\Phi_{a}^{1} y\right)(t)\right\| \\
& \leq \| \sum_{k=1}^{m} T_{\alpha}\left(t-t_{k}\right)\left(t-t_{k}\right)^{\alpha-1} I_{k}\left(x\left(t_{k}^{-}\right)\right) \\
& \quad-\sum_{k=1}^{m} T_{\alpha}\left(t-t_{k}\right)\left(t-t_{k}\right)^{\alpha-1} I_{k}\left(y\left(t_{k}^{-}\right)\right) \| \\
& \leq \frac{M}{\Gamma(\alpha)} \sum_{k=1}^{m} d_{k}\|x-y\| .
\end{aligned}
$$

Then, $\Phi_{a}^{1}$ is a contraction operator, since $(M / \Gamma(\alpha)) \sum_{k=1}^{m} d_{k}<$ 1.

Next, we prove that $\Phi_{a}^{2}$ is u.s.c and completely continuous. We subdivide the proof into several claims.

Claim 1. There exists a positive constant $r$ such that $\Phi_{a}^{2}\left(B_{r}\right) \subseteq$ $B_{r}$.

By employing the technique used in Step 2, one can easily show that there exists $r>0$ such that $\Phi_{a}^{2}\left(B_{r}\right) \subseteq B_{r}$.

Claim 2. $\Phi_{a}^{2}\left(B_{r}\right)$ is a family of equicontinuous functions.

Let $0 \leq s \leq t_{1} \leq t_{2} \leq b$. For each $x \in B_{r}, \varphi \in \Phi_{a}^{2}(x)$, there exists $f \in S_{F, x}$ such that

$$
\begin{aligned}
\tau(t)= & t^{\alpha-1} T_{\alpha}(t) x_{0} \\
& +\int_{0}^{t}(t-s)^{\alpha-1} T_{\alpha}(t-s) f(s) d s \\
& +\int_{0}^{t}(t-s)^{\alpha-1} T_{\alpha}(t-s) B u(s) d s, \quad t \in J .
\end{aligned}
$$


Then, we have

$$
\begin{aligned}
& \left\|\tau\left(t_{2}\right)-\tau\left(t_{1}\right)\right\| \\
& \leq\left\|\int_{0}^{t_{1}}\left[\left(t_{2}-s\right)^{\alpha-1}-\left(t_{1}-s\right)^{\alpha-1}\right] T_{\alpha}\left(t_{2}-s\right) f(s) d s\right\| \\
& \quad \times\left\|\int_{0}^{t_{1}}\left(t_{1}-s\right)^{\alpha-1}\left[T_{\alpha}\left(t_{2}-s\right)-T_{\alpha}\left(t_{1}-s\right)\right] f(s) d s\right\| \\
& \quad+\left\|\int_{t_{1}}^{t_{2}}\left(t_{2}-s\right)^{\alpha-1} T_{\alpha}\left(t_{2}-s\right) f(s) d s\right\| \\
& \quad \times\left\|\int_{0}^{t_{1}}\left[\left(t_{2}-s\right)^{\alpha-1}-\left(t_{1}-s\right)^{\alpha-1}\right] T_{\alpha}\left(t_{2}-s\right) u(s) d s\right\| \\
& \quad \times\left\|\int_{0}^{t_{1}}\left(t_{1}-s\right)^{\alpha-1}\left[T_{\alpha}\left(t_{2}-s\right)-T_{\alpha}\left(t_{1}-s\right)\right] u(s) d s\right\| \\
& \quad+\left\|\int_{t_{1}}^{t_{2}}\left(t_{2}-s\right)^{\alpha-1} T_{\alpha}\left(t_{2}-s\right) u(s) d s\right\| \\
& \leq I_{1}+I_{2}+I_{3}+I_{4}+I_{5}+I_{6} .
\end{aligned}
$$

By using Hölder's inequality and assumption $H(3)$, we get

$$
\begin{gathered}
I_{1}=\frac{M \psi(r)\|P\|_{L^{1 / \gamma}}}{\Gamma(\alpha)} \\
\times\left[\left(\frac{1-\gamma}{\alpha-\gamma} t_{1}^{(\alpha-\gamma) /(1-\gamma)}\right)^{1-\gamma}\right. \\
+\left(\frac{1-\gamma}{\alpha-\gamma}\left(t_{2}-t_{1}\right)^{(\alpha-\gamma) /(1-\gamma)}\right)^{1-\gamma} \\
\left.I_{2}=\psi\left(\frac{1-\gamma}{\alpha-\gamma} t_{2}^{(\alpha-\gamma) /(1-\gamma)}\right)^{1-\gamma}\right], \\
I_{6}=\frac{M \|_{L^{1 / \gamma}}\left(\frac{1-\gamma}{\alpha-\gamma} t_{1}^{(\alpha-\gamma) /(1-\gamma)}\right)^{1-\gamma}}{\Gamma(\alpha)} \int_{t_{1}}^{\sup _{s}\left[0, t_{1}\right]}\left\|T_{\alpha}\left(t_{2}-s\right)-T_{\alpha}\left(t_{1}-s\right)\right\|, \\
I_{3}=\frac{M \psi(r)\|P\|_{L^{1 / \gamma}}}{\Gamma(\alpha)}\left[\frac{1-\gamma}{\alpha-\gamma}\left(t_{2}-t_{1}\right)^{(\alpha-\gamma) /(1-\gamma)}\right]^{1-\gamma}, \\
I_{4}=\frac{M M_{B}}{\Gamma(\alpha)} \int_{t_{1}}^{t_{2}}\left[\left(t_{1}-s\right)^{\alpha-1}\|u(s)\| d s,\right. \\
I_{5} \sup _{2}\left\|T_{\alpha}\left(t_{2}-s\right)-T_{\alpha}\left(t_{1}-s\right)\right\|
\end{gathered}
$$

It is easy to see that $I_{2}$ tends to zero independently of $x \in B_{r}$ as $t_{2} \rightarrow t_{1}$. Note that, from Lemma 10, $T_{\alpha}(t)$ is continuous in the uniform operator topology for $t>0$; we can directly obtain $I_{1}$ and $I_{3}$ tending to zero independently of $x \in B_{r}$ as $t_{2} \rightarrow t_{1}$. Applying the absolute continuity of the Lebesgue integral, we have $I_{4}, I_{5}$, and $I_{6}$ tending to zero independently of $x \in B_{r}$ as $t_{2} \rightarrow t_{1}$.

Therefore, $\Phi_{a}^{2}\left(B_{r}\right) \subset P C_{1-\alpha}(J, X)$ is equicontinuous.

Claim 3. The set $\Pi(t)=\left\{\tau(t): \tau \in \Phi_{a}^{2}\left(B_{r}\right)\right\} \subset X$ is relatively compact for each $t \in J$.

Let $0<t \leq b$ be fixed. For $x \in B_{r}$ and $\tau \in \Phi_{a}^{2}(x)$, there exists $f \in S_{F, x}$ such that, for each $t \in J$,

$$
\begin{gathered}
\tau(t)=t^{\alpha-1} T_{\alpha}(t) x_{0}+\int_{0}^{t}(t-s)^{\alpha-1} T_{\alpha}(t-s) f(s) d s \\
+\int_{0}^{t}(t-s)^{\alpha-1} T_{\alpha}(t-s) B B^{*} T_{\alpha}^{*}(b-t) R\left(a, \Gamma_{0}^{b}\right) \\
\times\left[x_{1}-b^{\alpha-1} T_{\alpha}(b) x_{0}\right. \\
\quad-\sum_{k=1}^{m} T_{\alpha}\left(b-t_{k}\right)\left(b-t_{k}\right)^{\alpha-1} I_{k}\left(x\left(t_{k}^{-}\right)\right) \\
\left.\quad-\int_{0}^{b}(b-\mu)^{\alpha-1} T_{\alpha}(b-\mu) f(\mu) d \mu\right] d s
\end{gathered}
$$

For all $\varepsilon \in(0, t)$ and for all $\delta>0$, define

$$
\begin{aligned}
& \tau^{\varepsilon, \delta}(t) \\
& =t^{\alpha-1} T_{\alpha}(t) x_{0} \\
& +\alpha \int_{0}^{t-\varepsilon} \int_{\delta}^{\infty} \theta(t-s)^{\alpha-1} \xi_{\alpha}(\theta) T\left((t-s)^{\alpha} \theta\right) f(s) d \theta d s \\
& +\alpha \int_{0}^{t-\varepsilon} \int_{\delta}^{\infty} \theta(t-s)^{\alpha-1} \xi_{\alpha}(\theta) T\left((t-s)^{\alpha} \theta\right) \\
& \times B B^{*} T_{\alpha}^{*}(b-s) R\left(a, \Gamma_{0}^{b}\right) \\
& \times\left[x_{1}-b^{\alpha-1} T_{\alpha}(b) x_{0}\right. \\
& -\sum_{k=1}^{m} T_{\alpha}\left(b-t_{k}\right)\left(b-t_{k}\right)^{\alpha-1} I_{k}\left(x\left(t_{k}^{-}\right)\right) \\
& \left.-\int_{0}^{b}(b-\mu)^{\alpha-1} T_{\alpha}(b-\mu) f(\mu) d \mu\right] d \theta d s \\
& =t^{\alpha-1} T_{\alpha}(t) x_{0}+T\left(\varepsilon^{\alpha} \delta\right) \\
& \times\left\{\alpha \int_{0}^{t-\varepsilon} \int_{\delta}^{\infty} \theta(t-s)^{\alpha-1} \xi_{\alpha}(\theta) T\right. \\
& \times\left((t-s)^{\alpha} \theta-\varepsilon^{\alpha} \delta\right) f(s) d \theta d s
\end{aligned}
$$




$$
\begin{aligned}
+\alpha \int_{0}^{t-\varepsilon} \int_{\delta}^{\infty} \theta(t-s)^{\alpha-1} \xi_{\alpha}(\theta) T\left((t-s)^{\alpha} \theta-\varepsilon^{\alpha} \delta\right) \\
\quad \times B B^{*} T_{\alpha}^{*}(b-s) R\left(a, \Gamma_{0}^{b}\right) \\
\times\left[x_{1}-b^{\alpha-1} T_{\alpha}(b) x_{0}\right. \\
\quad-\sum_{k=1}^{m} T_{\alpha}\left(b-t_{k}\right)\left(b-t_{k}\right)^{\alpha-1} I_{k}\left(x\left(t_{k}^{-}\right)\right) \\
\left.\left.\quad-\int_{0}^{b}(b-\mu)^{\alpha-1} T_{\alpha}(b-\mu) f(\mu) d \mu\right] d \theta d s\right\} .
\end{aligned}
$$

By the compactness of $T\left(\varepsilon^{\alpha} \delta\right)\left(\varepsilon^{\alpha} \delta>0\right)$, we obtain the set

$$
\Pi^{\varepsilon, \delta}(t)=\left\{\varphi^{\varepsilon, \delta}(t): \tau \in \Phi_{a}^{2}\left(B_{r}\right)\right\}
$$

which is relatively compact in $X$ for all $\varepsilon \in(0, t)$ and $\delta>0$. Moreover, we have

$$
\begin{aligned}
& \left\|\tau(t)-\tau^{\varepsilon, \delta}(t)\right\| \\
& \leq\left\{\frac{\alpha M \psi(r) \beta}{\Gamma(\alpha)}\|P\|_{L^{1 / \gamma}}+\frac{\alpha M^{2} M_{B}^{2} b^{2 \alpha-1}}{a(2 \alpha-1) \Gamma(\alpha)}\right. \\
& \quad \times\left[\left\|x_{1}\right\|+\frac{M\left\|x_{0}\right\|}{\Gamma(\alpha)}\right. \\
& \quad+\sum_{k=1}^{m} \frac{M}{\Gamma(\alpha)}\left(d_{k}\|x\|+m\left(b-t_{k}\right)^{\alpha-1}\left\|I_{k}(0)\right\|\right) \\
& \left.\left.+\frac{M \psi(r) \beta}{\Gamma(\alpha)}\|P\|_{L^{1 / \gamma}}\right]\right\} \int_{0}^{\delta} \theta \xi_{\alpha}(\theta) d \theta \\
& +\frac{M \psi(r)}{\Gamma(\alpha)}\|P\|_{L^{1 / \gamma}}\left(\frac{1-\gamma}{\alpha-\gamma} \varepsilon^{(\alpha-\gamma) /(1-\gamma)}\right)^{1-\gamma} \\
& +\frac{M^{2} M_{B}^{2} \varepsilon^{2 \alpha-1}}{a(2 \alpha-1)(\Gamma(\alpha))^{2}} \\
& \quad\left[\left\|x_{1}\right\|+\frac{M\left\|x_{0}\right\|}{\Gamma(\alpha)}\right. \\
& +\sum_{k=1}^{m} \frac{M}{\Gamma(\alpha)}\left(d_{k}\|x\|+m\left(b-t_{k}\right)^{\alpha-1}\left\|I_{k}(0)\right\|\right) \\
& \left.+\frac{M \psi(r) \beta}{\Gamma(\alpha)}\|P\|_{L^{1 / \gamma}}\right] .
\end{aligned}
$$

The right-hand side of the above inequality tends to zero as $\varepsilon \rightarrow 0$. Therefore, there are relatively compact sets arbitrarily close to the set $\Pi(t), t>0$. Hence the set $\Pi(t), t>0$ is also relatively compact in $X$. As a consequence of Claims 13 together with the Arzola-Ascoli theorem, we can conclude that $\Phi_{a}^{2}$ is completely continuous.
Claim 4. $\Phi_{\alpha}^{2}$ has a closed graph.

Let $x_{n} \rightarrow x^{*}(n \rightarrow \infty), \tau_{n} \in \Phi_{a}^{2}\left(x_{n}\right), \tau_{n} \rightarrow \tau^{*}(n \rightarrow$ $\infty)$. We will prove that $\tau^{*} \in \Phi_{a}^{2}\left(x^{*}\right)$. Since $\tau_{n} \in \Phi_{a}^{2}\left(x_{n}\right)$, there exists $f_{n} \in S_{F, x_{n}}$, such that, for each $t \in J$,

$$
\begin{aligned}
\tau_{n}(t)= & t^{\alpha-1} T_{\alpha}(t) x_{0} \\
& +\int_{0}^{t}(t-s)^{\alpha-1} T_{\alpha}(t-s) f_{n}(s) d s \\
& +\int_{0}^{t}(t-s)^{\alpha-1} T_{\alpha}(t-s) B B^{*} T_{\alpha}^{*}(b-t) R\left(a, \Gamma_{0}^{b}\right) \\
& \times\left[x_{1}-b^{\alpha-1} T_{\alpha}(b) x_{0}\right. \\
& \quad-\sum_{k=1}^{m} T_{\alpha}\left(b-t_{k}\right)\left(b-t_{k}\right)^{\alpha-1} I_{k}\left(x\left(t_{k}^{-}\right)\right) \\
& \left.\quad-\int_{0}^{b}(b-\mu)^{\alpha-1} T_{\alpha}(b-\mu) f_{n}(\mu) d \mu\right] d s .
\end{aligned}
$$

We must prove that there exists $f^{*} \in S_{F, x^{*}}$, such that, for each $t \in J$,

$$
\begin{aligned}
\tau^{*}(t)= & t^{\alpha-1} T_{\alpha}(t) x_{0} \\
& +\int_{0}^{t}(t-s)^{\alpha-1} T_{\alpha}(t-s) f^{*}(s) d s \\
& +\int_{0}^{t}(t-s)^{\alpha-1} T_{\alpha}(t-s) B B^{*} T_{\alpha}^{*}(b-t) R\left(a, \Gamma_{0}^{b}\right) \\
& \times\left[x_{1}-b^{\alpha-1} T_{\alpha}(b) x_{0}\right. \\
& \quad-\sum_{k=1}^{m} T_{\alpha}\left(b-t_{k}\right)\left(b-t_{k}\right)^{\alpha-1} I_{k}\left(x\left(t_{k}^{-}\right)\right) \\
& \left.\quad-\int_{0}^{b}(b-\mu)^{\alpha-1} T_{\alpha}(b-\mu) f^{*}(\mu) d \mu\right] d s .
\end{aligned}
$$

Since $\tau_{n} \rightarrow \tau^{*}(n \rightarrow \infty)$, we can obtain

$$
\begin{aligned}
& \|\left(\tau_{n}(t)-t^{\alpha-1} T_{\alpha}(t) x_{0}\right. \\
& -\int_{0}^{t}(t-s)^{\alpha-1} T_{\alpha}(t-s) B B^{*} T_{\alpha}^{*}(b-t) R\left(a, \Gamma_{0}^{b}\right) \\
& \quad \times\left[x_{1}-b^{\alpha-1} T_{\alpha}(b) x_{0}\right. \\
& \left.\left.\quad-\sum_{k=1}^{m} T_{\alpha}\left(b-t_{k}\right)\left(b-t_{k}\right)^{\alpha-1} I_{k}\left(x\left(t_{k}^{-}\right)\right)\right] d s\right)
\end{aligned}
$$




$$
\begin{gathered}
-\left(\tau^{*}(t)-t^{\alpha-1} T_{\alpha}(t) x_{0}\right. \\
-\int_{0}^{t}(t-s)^{\alpha-1} T_{\alpha}(t-s) B B^{*} T_{\alpha}^{*}(b-t) R\left(a, \Gamma_{0}^{b}\right) \\
\times\left[x_{1}-b^{\alpha-1} T_{\alpha}(b) x_{0}\right. \\
\quad-\sum_{k=1}^{m} T_{\alpha}\left(b-t_{k}\right)\left(b-t_{k}\right)^{\alpha-1} \\
\left.\left.\times I_{k}\left(x\left(t_{k}^{-}\right)\right)\right] d s\right) \| 0, \quad \text { as } n \longrightarrow \infty .
\end{gathered}
$$

Consider the linear continuous operator

$$
\begin{gathered}
\Gamma: L^{1 / \gamma}(J, X) \longrightarrow C_{1-\alpha}(J, X) \\
(\Gamma f)(t)=\int_{0}^{t}(t-s)^{\alpha-1} T_{\alpha}(t-s) \\
\times\left[f(s)-B(b-s)^{\alpha-1} B^{*} T_{\alpha}^{*}(b-s) R\left(a, \Gamma_{0}^{b}\right)\right. \\
\left.\quad \times \int_{0}^{b} T_{\alpha}(b-\eta) f(\eta) d \eta\right] d s .
\end{gathered}
$$

Clearly it follows from Lemma 13 that $\Gamma \circ S_{F}$ is a closed graph operator. Moreover, we have

$$
\begin{aligned}
& \tau_{n}(t)-t^{\alpha-1} T_{\alpha}(t) x_{0} \\
& -\int_{0}^{t}(t-s)^{\alpha-1} T_{\alpha}(t-s) B B^{*} T_{\alpha}^{*}(b-s) R\left(a, \Gamma_{0}^{b}\right) \\
& \quad \times\left[x_{1}-b^{\alpha-1} T_{\alpha}(b) x_{0}\right. \\
& \left.\quad-\sum_{k=1}^{m} T_{\alpha}\left(b-t_{k}\right)\left(b-t_{k}\right)^{\alpha-1} I_{k}\left(x\left(t_{k}^{-}\right)\right)\right] d s \in \Gamma\left(S_{F, x_{n}}\right) .
\end{aligned}
$$

Since $x_{n} \rightarrow x_{*}$, it follows from Lemma 13 that

$$
\begin{aligned}
& \tau_{*}(t)-t^{\alpha-1} T_{\alpha}(t) x_{0} \\
& -\int_{0}^{t}(t-s)^{\alpha-1} T_{\alpha}(t-s) B B^{*} T_{\alpha}^{*}(b-s) R\left(a, \Gamma_{0}^{b}\right) \\
& \quad \times\left[x_{1}-b^{\alpha-1} T_{\alpha}(b) x_{0}\right. \\
& \left.\quad-\sum_{k=1}^{m} T_{\alpha}\left(b-t_{k}\right)\left(b-t_{k}\right)^{\alpha-1} I_{k}\left(x\left(t_{k}^{-}\right)\right)\right] d s \in \Gamma\left(S_{F, x_{*}}\right) .
\end{aligned}
$$

Therefore, $\Phi_{a}^{2}$ has a closed graph. Since $\Phi_{a}^{2}$ is a completely continuous multivalued map with compact value, we have that $\Phi_{a}^{2}$ is u.s.c.

Thus $\Phi_{a}=\Phi_{a}^{1}+\Phi_{a}^{2}$ is u.s.c and condensing. Therefore, applying Lemma 13, we conclude that $\Phi_{a}$ has a fixed point $x(\cdot)$ on $B_{r_{0}}$. Thus, the fractional control system (4) has a mild solution on $J$.

The proof is complete.

The following result concerns the approximate controllability of that problem (4). We assume that the following assumption be held.

$H^{\prime}(3)$ : There exists a positive constant $L$ such that $\|F(t, x(t))\| \leq L$ for all $(t, x) \in J \times X$.

Theorem 15. Assume that assumptions $H(1), H(2), H^{\prime}(3)$, and $H(4)$ are satisfied and the linear system (24) is approximately controllable on J. Then system (4) is approximately controllable on $\mathrm{J}$.

Proof. By employing the technique used in Theorem 14, we can easily show that, for all $0<a<1$, the operator $\Phi_{a}$ has a fixed point in $B_{r_{0}}$, where $r_{0}=r(a)$. Let $x^{a}(\cdot)$ be a fixed point of $\Phi_{a}$ in $B_{r_{0}}$. Any fixed point of $\Phi_{a}$ is a mild solution of (4); this means that there exists $f^{a} \in S_{F, x^{a}}$ such that, for each $t \in J^{\prime}$,

$$
\begin{aligned}
& x^{a}(t) \\
& =t^{\alpha-1} T_{\alpha}(t) x_{0} \\
& +\sum_{k=1}^{m} T_{\alpha}\left(t-t_{k}\right)\left(t-t_{k}\right)^{\alpha-1} I_{k}\left(x\left(t_{k}^{-}\right)\right) \\
& +\int_{0}^{t}(t-s)^{\alpha-1} T_{\alpha}(t-s) f^{a}(s) d s \\
& +\int_{0}^{t}(t-s)^{\alpha-1} T_{\alpha}(t-s) B B^{*} T_{\alpha}^{*}(b-s) R\left(a, \Gamma_{0}^{b}\right) \\
& \times\left[x_{1}-b^{\alpha-1} T_{\alpha}(b) x_{0}\right. \\
& \quad-\sum_{k=1}^{m} T_{\alpha}\left(b-t_{k}\right)\left(b-t_{k}\right)^{\alpha-1} I_{k}\left(x\left(t_{k}^{-}\right)\right) \\
& \left.\quad-\int_{0}^{b}(b-\mu)^{\alpha-1} T_{\alpha}(b-\mu) f^{a}(\mu) d \mu\right] d s .
\end{aligned}
$$

Define

$$
\begin{aligned}
& P\left(f^{a}\right) \\
& =x_{1}-b^{\alpha-1} T_{\alpha}(b) x_{0} \\
& -\sum_{k=1}^{m} T_{\alpha}\left(b-t_{k}\right)\left(b-t_{k}\right)^{\alpha-1} I_{k}\left(x\left(t_{k}^{-}\right)\right)
\end{aligned}
$$




$$
\begin{array}{r}
-\int_{0}^{b}(b-s)^{\alpha-1} T_{\alpha}(b-s) f^{a}(s) d s \\
\text { for some } f^{a} \in S_{F, x^{a}} .
\end{array}
$$

Noting that $I-\Gamma_{0}^{b} R\left(a, \Gamma_{0}^{b}\right)=a R\left(a, \Gamma_{0}^{b}\right)$, we get

$$
x^{a}(b)=x_{1}-a R\left(a, \Gamma_{0}^{b}\right) P\left(f^{a}\right) \text {. }
$$

By assumption $H^{\prime}(3)$,

$$
\int_{0}^{b}\left\|f^{a}(s)\right\|^{2} d s \leq L^{2} b .
$$

Consequently the sequence $\left\{f^{a}\right\}$ is uniformly bounded in $L^{1 / \gamma}(J, X)$. Thus, there is a subsequence, still denoted by $\left\{f^{a}\right\}$, that converges weakly to, say, $f$ in $L^{1 / \gamma}(J, X)$. Denoting

$$
\begin{aligned}
h= & x_{1}-b^{\alpha-1} T_{\alpha}(b) x_{0} \\
& -\sum_{k=1}^{m} T_{\alpha}\left(b-t_{k}\right)\left(b-t_{k}\right)^{\alpha-1} I_{k}\left(x\left(t_{k}^{-}\right)\right) \\
& -\int_{0}^{b}(b-s)^{\alpha-1} T_{\alpha}(b-s) f(s) d s,
\end{aligned}
$$

we see that

$$
\begin{aligned}
& \left\|P\left(f^{\alpha}\right)-h\right\| \\
& \quad=\left\|\int_{0}^{b}(b-s)^{\alpha-1} T_{\alpha}(b-s)\left[f^{a}(s)-f(s)\right] d s\right\| \\
& \quad \leq \sup _{0 \leq t \leq b}\left\|\int_{0}^{b}(b-s)^{\alpha-1} T_{\alpha}(b-s)\left[f^{\alpha}(s)-f(s)\right] d s\right\| .
\end{aligned}
$$

By the Ascoli-Arzela theorem, we can show that the linear operator $g \rightarrow \int_{0}^{\cdot}(\cdot-s)^{\alpha-1} T_{\alpha}(\cdot-s) g(s) d s: L^{1 / \gamma}(J, X) \rightarrow$ $P C_{1-\alpha}(J, X)$ is compact; consequently the right-hand side of (93) tends to zero as $a \rightarrow 0^{+}$.

This implies that

$$
\begin{aligned}
& \left\|x^{a}(b)-x_{1}\right\| \\
& \quad=\left\|a R\left(a, \Gamma_{0}^{b}\right) P\left(f^{a}\right)\right\| \\
& \quad \leq\left\|a R\left(a, \Gamma_{0}^{b}\right)(h)\right\|+\left\|a R\left(\alpha, \Gamma_{0}^{b}\right)\left(P\left(f^{a}\right)-h\right)\right\| \\
& \quad \leq\left\|a R\left(a, \Gamma_{0}^{b}\right)(h)\right\|+\left\|P\left(f^{a}\right)-h\right\| \longrightarrow 0, \quad \text { as } a \longrightarrow 0^{+} .
\end{aligned}
$$

This proves the approximate controllability of system (4).

Remark 16. In [9], Ganesh et al. have studied the approximate controllability of fractional integrodifferential evolution equations. If our problem (4) can be changed as

$$
\begin{gathered}
D_{t}^{\alpha} x(t) \in A x(t)+B u(t)+F(t, x(t),(H x)(t)), \\
t \in(0, b], \quad \frac{1}{2}<\alpha \leq 1 \\
\left.I_{0^{+}}^{1-\alpha} x(t)\right|_{t=0}=x_{0}+g(x) \in X
\end{gathered}
$$

where $D_{t}^{\alpha}$ denotes the Riemann-Liouville fractional derivative, the operator $H$ is defined by $(H x)(t)=\int_{0}^{b} h(t, s, x(s)) d s$. By a similar way, we can get the approximate controllability of the system (95).

Remark 17. In [15], Rathinasamy and Yong have researched the approximate controllability of fractional differential equations with state-dependent delay. Our problem (4) can be adopted to the impulsive fractional differential inclusion system with state-dependent delay:

$$
\begin{gathered}
D_{t}^{\alpha} x(t) \in A x(t)+B u(t)+F\left(t, x_{\rho\left(t, x_{t}\right)}\right), \\
t \in(0, b], \quad \frac{1}{2}<\alpha \leq 1, \\
\left.\Delta I_{0^{+}}^{1-\alpha} x(t)\right|_{t=t_{k}}=I_{k}\left(x\left(t_{k}^{-}\right)\right), \quad k=1,2, \ldots, m, \\
\left.I_{0^{+}}^{1-\alpha} x(t)\right|_{t=0}=\phi \in X,
\end{gathered}
$$

where $D_{t}^{\alpha}$ is the Riemann-Liouville fractional derivative, $A$ is the infinitesimal generator of a $C_{0}$-semigroup $\{T(t), t \geq 0\}$ on a Banach space $X$, the state $x(\cdot)$ takes values in $X$, the control function $u(\cdot)$ is given in $L^{p}(J, U)(p>1 / \alpha), U$ is a Banach space of admissible control, and $B: U \rightarrow X$ is a bounded linear operator. $F: J \times D \rightarrow \mathscr{P}(X):=2^{X} \backslash\{\emptyset\}$ with $D:=C((-\infty, 0], X)$ is a multivalued map. For a continuous function $x: J^{*}:=(-\infty, b] \rightarrow X, x_{t}$ is the element of $D$ defined by $x_{t}(s)=x(t+s),-\infty \leq s \leq 0$, and $\phi \in D$. Further, $\rho:[0, b] \times \mathscr{P}(X) \rightarrow(-\infty, b]$ are appropriate nonlinear functions.

\section{An Example}

In this final section, we give an example to illustrate our abstract results.

Example 1. Consider the following fractional partial differential inclusion with control

$$
\begin{gathered}
D_{t}^{\alpha} x(t, y) \in \frac{\partial^{2}}{\partial y^{2}} x(t, y)+F(t, x(t, y))+B u(t, y), \\
t \in J^{\prime}=[0,1] \backslash\left\{\frac{1}{2}\right\}, \quad y \in[0, \pi], \\
\Delta I_{0^{+}}^{1-\alpha} x\left(\frac{1}{2}, y\right)=\frac{|x(y)|}{2 \pi+|x(y)|}, \quad y \in[0, \pi], \\
x(t, 0)=x(t, \pi)=0, \quad t \in J=[0,1], \\
\left.I_{0^{+}}^{1-\alpha} x(t, y)\right|_{t=0}=x_{0}(y), \quad t \in[0,1], y \in[0, \pi],
\end{gathered}
$$

where $D^{\alpha}$ is the Riemann-Liouville fractional partial derivative of order $3 / 5, J=[0,1]$.

Let $X=U=L^{2}[0, \pi]$, define; the operator $A$ by $A x=x_{y y}$ with the domain

$$
\begin{gathered}
D(A)=\left\{x \in X: x, x^{\prime}\right. \text { are absolutely continuous, } \\
\left.x_{y y} \in X, x(t, 0)=x(t, \pi)=0\right\}
\end{gathered}
$$


Then,

$$
A x=-\sum_{n=1}^{\infty} n^{2}\left\langle x, e_{n}\right\rangle e_{n}, \quad x \in D(A),
$$

where $e_{n}(y)=\sqrt{2 / \pi} \sin n y, 0 \leq y \leq \pi, n=1,2, \ldots$, is the orthogonal set of eigenvectors of $A$. It is easily shown that operator $A$ generates a strongly continuous semigroup $\{T(t): t \geq 0\}$ on $X$, which are compact, analytic, and selfadjoint in $X$.

It can be easily seen that the linear system corresponding to (97) is approximately controllable on $[0,1][42]$.

Define $x(t)(y)=x(t, y)$; let $B: U \rightarrow X$ be defined by $B u(t)(y)=B u(t, y)$. Then system (97) can be written in the abstract form given by (4). We assume that $F: J \times D \rightarrow \mathscr{P}(X)$ satisfy the following conditions.

$\left(F_{1}\right): F: J \times D \rightarrow \mathscr{P}_{c p, c v}(X)$ is measurable to $t$ for each fixed $x \in D$, u.s.c. to $x$ for a.e. $t \in J$, and for each $x \in P C_{1-\alpha}(J, X)$ the set

$$
S_{F, x}=\left\{f \in L^{1}(J, X): f(t) \in F(t, x)\right\}
$$

is nonempty.

$\left(F_{2}\right)$ : There exists a positive constant $L$ such that $\|F(t, x)\| \leq L$ for all $(t, x) \in J \times D$, since, for any $x, y \in A C(J, X)$, we have

$$
\left\|\Delta I_{t}^{1-\alpha} x\left(t_{k}, z\right)-\Delta I_{t}^{1-\alpha} y\left(t_{k}, z\right)\right\| \leq \frac{\|x-y\|}{2} .
$$

Obviously, the hypotheses of Theorem 15 are fulfilled. Thus, the system (97) is approximately controllable on $[0,1]$.

\section{Conclusions}

In this paper, we study the approximate controllability for impulsive fractional differential inclusions with RiemannLiouville fractional derivatives in Banach spaces. We mainly use fixed point theorem and semigroup theory; we obtain some new mild solutions for this kind of problems (4). An illustrative example is also discussed to show the effectiveness of the results in this paper. In the near future, we will consider Riemann-Liouville fractional partial differential problems, which will be more complicated.

\section{Acknowledgments}

The project was supported by NNSF of China Grant nos. 11271087 and 61263006, the Scientific Research Foundation of Guangxi University for Nationalities no. 2012QD014, and the Innovation Project of Guangxi University for Nationalities no. gxun-chx2013083.

\section{References}

[1] R. E. Kalman, "Controllability of linear dynamical systems," Contributions to Differential Equations, vol. 1, pp. 190-213, 1963.
[2] N. Abada, M. Benchohra, and H. Hammouche, "Existence and controllability results for nondensely defined impulsive semilinear functional differential inclusions," Journal of Differential Equations, vol. 246, no. 10, pp. 3834-3863, 2009.

[3] A. E. Bashirov and N. I. Mahmudov, "On concepts of controllability for deterministic and stochastic systems," SIAM Journal on Control and Optimization, vol. 37, no. 6, pp. 1808-1821, 1999.

[4] X. Fu, "Controllability of non-densely defined functional differential systems in abstract space," Applied Mathematics Letters, vol. 19, no. 4, pp. 369-377, 2006.

[5] M. Benchohra and A. Ouahab, "Controllability results for functional semilinear differential inclusions in Fréchet spaces," Nonlinear Analysis: Theory, Methods \& Applications, vol. 61, no. 3, pp. 405-423, 2005.

[6] Y.-K. Chang and D. N. Chalishajar, "Controllability of mixed Volterra-Fredholm-type integro-differential inclusions in Banach spaces," Journal of the Franklin Institute, vol. 345, no. 5, pp. 499-507, 2008.

[7] L. Górniewicz, S. K. Ntouyas, and D. O'Regan, "Controllability of semilinear differential equations and inclusions via semigroup theory in Banach spaces," Reports on Mathematical Physics, vol. 56, no. 3, pp. 437-470, 2005.

[8] R. Triggiani, "A note on the lack of exact controllability for mild solutions in Banach spaces," SIAM Journal on Control and Optimization, vol. 15, no. 3, pp. 407-411, 1977.

[9] R. Ganesh, R. Sakthivel, N. I. Mahmudov, and S. M. Anthoni, "Approximate controllability of fractional integrodifferential evolution equations," Journal of Applied Mathematics, vol. 2013, Article ID 291816, 7 pages, 2013.

[10] Z. Liu, J. Y. Lv, and R. Sakthivel, "Approximate controllability of fractional functional evolution inclusions with delay in Hilbert spaces," IMA Journal of Mathematical Control and Information, 2013.

[11] Z. Liu, "Browder-Tikhonov regularization of non-coercive evolution hemivariational inequalities," Inverse Problems, vol. 21, no. 1, pp. 13-20, 2005.

[12] Z. Liu, "Existence results for quasilinear parabolic hemivariational inequalities," Journal of Differential Equations, vol. 244, no. 6, pp. 1395-1409, 2008.

[13] R. Sakthivel, Y. Ren, and N. I. Mahmudov, "On the approximate controllability of semilinear fractional differential systems," Computers \& Mathematics with Applications, vol. 62, no. 3, pp. 1451-1459, 2011.

[14] R. Sakthivel, R. Ganesh, Y. Ren, and S. M. Anthoni, "Approximate controllability of nonlinear fractional dynamical systems," Communications in Nonlinear Science and Numerical Simulation, vol. 18, no. 12, pp. 3498-3508, 2013.

[15] S. Rathinasamy and R. Yong, "Approximate controllability of fractional differential equations with state-dependent delay," Results in Mathematics, vol. 63, no. 3-4, pp. 949-963, 2013.

[16] N. Sukavanam and S. Kumar, "Approximate controllability of fractional order semilinear delay systems," Journal of Optimization Theory and Applications, vol. 151, no. 2, pp. 373-384, 2011.

[17] H. X. Zhou, "Approximate controllability for a class of semilinear abstract equations," Society for Industrial and Applied Mathematics, vol. 21, no. 4, pp. 551-565, 1983.

[18] K. Rykaczewski, "Approximate controllability of differential inclusions in Hilbert spaces," Nonlinear Analysis: Theory, Methods \& Applications, vol. 75, no. 5, pp. 2701-2712, 2012.

[19] D. Baleanu, A. Ranjbar N., S. J. Sadati R., H. Delavari, T. Abdeljawad, and V. Gejji, "Lyapunov-Krasovskii stability theorem for 
fractional systems with delay," Romanian Journal of Physics, vol. 56, no. 5-6, pp. 636-643, 2011.

[20] K. Diethelm and A. D. Freed, "On the solution of nonlinear fractional order differential equations used in the modeling of viscoplasticity," in Scientifice Computing in Chemical Engineering II-Computational Fluid Dynamics, Reaction Engineering and Molecular Properties, F. Keil, W. Mackens, H. Voss, and J. Werther, Eds., pp. 217-224, Springer, Heidelberg, Germany, 1999.

[21] A. D. Fitt, A. R. H. Goodwin, K. A. Ronaldson, and W. A. Wakeham, "A fractional differential equation for a MEMS viscometer used in the oil industry," Journal of Computational and Applied Mathematics, vol. 229, no. 2, pp. 373-381, 2009.

[22] R. Hilfer, Applications of Fractional Calculus in Physics, World Scientific, River Edge, NJ, USA, 2000.

[23] K. B. Oldham and J. Spanier, The Fractional Calculus: Theory and Applications of Differentiation and Integration to Arbitrary Order, Academic Press, New York, NY, USA, 1974.

[24] V. Lakshmikantham, S. Leela, and J. Vasundhara Devi, Theory of Fractional Dynamic Systems, Cambridge Academic, Cambridge, UK, 2009.

[25] J. Liang, Y. Liu, and Z. Liu, "A class of BVPS for first order impulsive integro-differential equations," Applied Mathematics and Computation, vol. 218, no. 7, pp. 3667-3672, 2011.

[26] Z. Liu, J. Han, and L. Fang, "Integral boundary value problems for first order integro-differential equations with impulsive integral conditions," Computers \& Mathematics with Applications, vol. 61, no. 10, pp. 3035-3043, 2011.

[27] J. Wang, M. Fečkan, and Y. Zhou, "On the new concept of solutions and existence results for impulsive fractional evolution equations," Dynamics of Partial Differential Equations, vol. 8, no. 4, pp. 345-361, 2011.

[28] A. A. Kilbas, H. M. Srivastava, and J. J. Trujillo, "Theory and applications of fractional differential equations," in NorthHolland Mathematics Studies, vol. 204, Elservier Science B.V., Amsterdam, The Netherlands, 2006.

[29] I. Podlubny, Fractional Differential Equations, vol. 198 of Mathematics in Science and Engineering, Academic Press, San Diego, Calif, USA, 1999.

[30] N. Heymans and I. Podlubny, "Physical interpretation of initial conditions for fractional differential equations with RiemannLiouville fractional derivatives," Rheologica Acta, vol. 45, no. 5, pp. 765-771, 2006.

[31] Y. Zhou and F. Jiao, "Existence of mild solutions for fractional neutral evolution equations," Computers \& Mathematics with Applications, vol. 59, no. 3, pp. 1063-1077, 2010.

[32] J. Cao and H. Chen, "Some results on impulsive boundary value problem for fractional differential inclusions," Electronic Journal of Qualitative Theory of Differential Equations, vol. 11, pp. 1-24, 2011.

[33] M. Benchohra, J. Henderson, and S. K. Ntouyas, "On nonresonance second order impulsive functional differential inclusions with nonlinear boundary conditions," Canadian Applied Mathematics Quarterly, vol. 14, no. 1, pp. 21-32, 2006.

[34] B. Ahmad and S. K. Ntouyas, "Boundary value problems for $n$-th order differential inclusions with four-point integral boundary conditions," Opuscula Mathematica, vol. 32, no. 2, pp. 205-226, 2012.

[35] M. Kisielewicz, Differential Inclusions and Optimal Control, vol. 44 of Mathematics and its Applications (East European Series), Kluwer Academic, Dordrecht, The Netherlands, 1991.
[36] J.-P. Aubin and A. Cellina, Differential Inclusions: Set-Valued Maps and Viability Theory, vol. 264, Springer, New York, NY, USA, 1984.

[37] K. Deimling, Multivalued Differential Equations, vol. 1 of de Gruyter Series in Nonlinear Analysis and Applications, Walter de Gruyter \& Co., Berlin, Germany, 1992.

[38] S. Hu and N. S. Papageorgiou, Handbook of Multivalued Analysis. Vol. I (Theory), vol. 419 of Mathematics and its Applications, Kluwer Academic, Dordrecht, The Netherlands, 1997.

[39] A. Lasota and Z. Opial, "An application of the Kakutani-Ky Fan theorem in the theory of ordinary differential equations," Bulletin de l'Académie Polonaise des Sciences, vol. 13, pp. 781-786, 1965.

[40] N. S. Papageorgiou, "On the theory of Banach space valued multifunctions. I. Integration and conditional expectation," Journal of Multivariate Analysis, vol. 17, no. 2, pp. 185-206, 1985.

[41] M. Kamenskii, V. Obukhovskii, and P. Zecca, Condensing Multivalued Maps and Semilinear Differential Inclusions in Banach Spaces, vol. 7 of De Gruyter Series in Nonlinear Analysis and Applications, Walter de Gruyter, Berlin, Germany, 2001.

[42] N. I. Mahmudov, "Controllability of semilinear stochastic systems in Hilbert spaces," Journal of Mathematical Analysis and Applications, vol. 288, no. 1, pp. 197-211, 2003. 


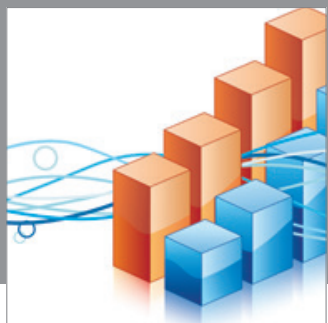

Advances in

Operations Research

mansans

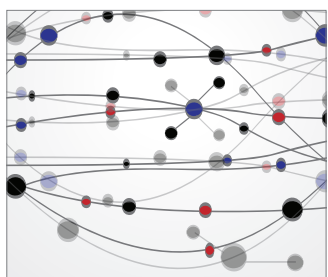

The Scientific World Journal
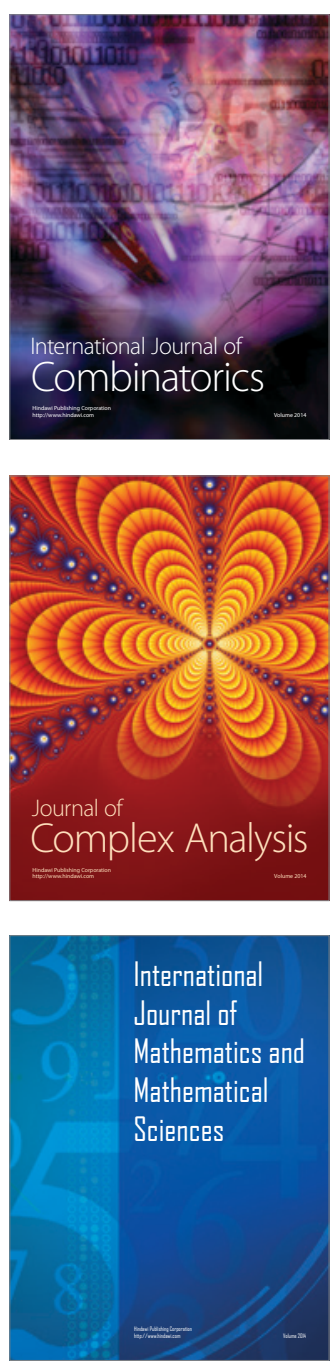
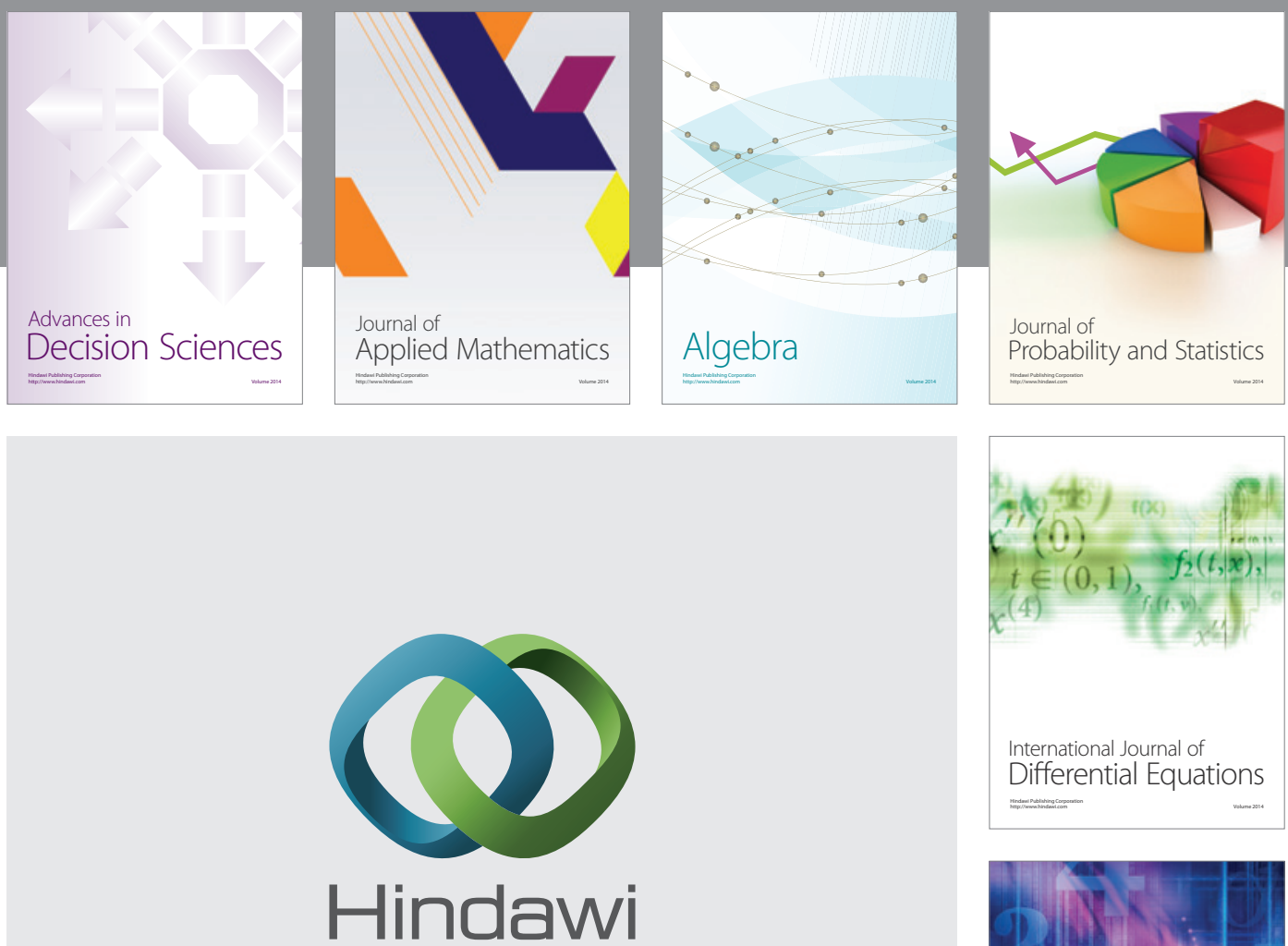

Submit your manuscripts at http://www.hindawi.com
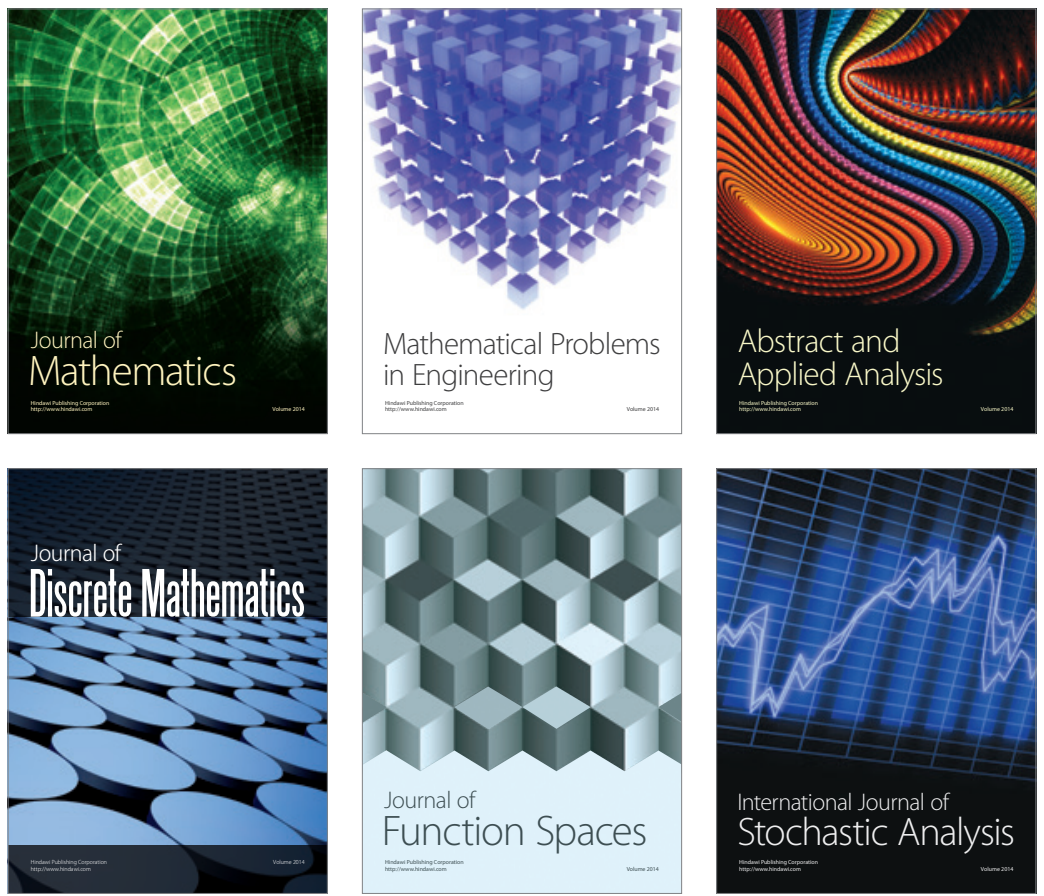

Journal of

Function Spaces

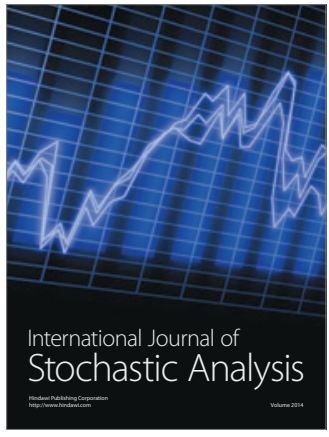

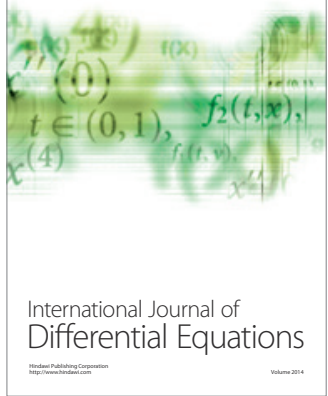
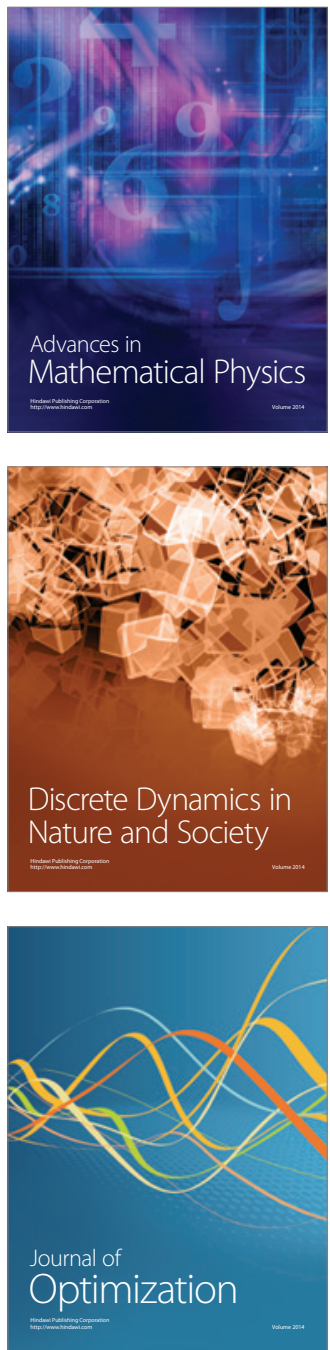